\title{
Global microRNA depletion suppresses tumor angiogenesis
}

\author{
Sidi Chen, ${ }^{1,2,6}$ Yuan Xue, ${ }^{1,6}$ Xuebing Wu ${ }^{1,3}$ Cong Le, ${ }^{2}$ Arjun Bhutkar, ${ }^{1}$ Eric L. Bell, ${ }^{4}$ Feng Zhang, ${ }^{2}$ \\ Robert Langer, ${ }^{1,5}$ and Phillip A. Sharp ${ }^{1,4,7}$ \\ ${ }^{1}$ Koch Institute for Integrative Cancer Research, Massachusetts Institute of Technology, Cambridge, Massachusetts 02139, USA; \\ ${ }^{2}$ Broad Institute of Massachusetts Institute of Technology and Harvard, Cambridge, Massachusetts 02142, USA; ${ }^{3}$ Computational \\ and Systems Biology Program, Massachusetts Institute of Technology, Cambridge, Massachusetts 02139, USA; ${ }^{4}$ Department of \\ Biology, Massachusetts Institute of Technology, Cambridge, Massachusetts 02139, USA; ${ }^{5}$ Department of Chemical Engineering, \\ Massachusetts Institute of Technology, Cambridge, Massachusetts 02139, USA
}

MicroRNAs delicately regulate the balance of angiogenesis. Here we show that depletion of all microRNAs suppresses tumor angiogenesis. We generated microRNA-deficient tumors by knocking out Dicer1. These tumors are highly hypoxic but poorly vascularized, suggestive of deficient angiogenesis signaling. Expression profiling revealed that angiogenesis genes were significantly down-regulated as a result of the microRNA deficiency. Factor inhibiting hypoxia-inducible factor 1 (HIF-1), FIH1, is derepressed under these conditions and suppresses HIF transcription. Knocking out FIH1 using CRISPR/Cas9-mediated genome engineering reversed the phenotypes of microRNA-deficient cells in HIF transcriptional activity, VEGF production, tumor hypoxia, and tumor angiogenesis. Using multiplexed CRISPR/Cas9, we deleted regions in FIH1 3' untranslated regions (UTRs) that contain microRNA-binding sites, which derepresses FIH1 protein and represses hypoxia response. These data suggest that microRNAs promote tumor responses to hypoxia and angiogenesis by repressing FIH1.

[Keywords: microRNA; Dicer; angiogenesis; hypoxia; CRISPR/Cas9; gene regulation]

Supplemental material is available for this article.

Received February 16, 2014; revised version accepted April 10, 2014.

MicroRNAs are a class of 20- to 22-nucleotide (nt) small RNAs that regulate diverse biological processes (Bartel 2009). The majority of genes in the mammalian genome are regulated by one or more microRNAs (Friedman et al. 2009; Ebert and Sharp 2012). Individual microRNAs and microRNA families have been reported to regulate multiple hallmarks of cancer, such as cell proliferation, apoptosis, metastasis, and angiogenesis, serving as oncogenes or tumor suppressor genes (He et al. 2005; Calin and Croce 2006).

MicroRNAs can serve as both positive and negative regulators of angiogenesis. For example, miR-15/16 and miR-221/222 suppress tumor-induced vasculature formation by targeting VEGF, c-kit, and eNOS mRNAs (Cimmino et al. 2005; Hua et al. 2006). On the other hand, miR-17-92, let-7, and miR-210 positively regulate tumor angiogenesis by inhibiting genes encoding endogenous angiogenesis inhibitors (Kuehbacher et al. 2007; Fasanaro et al. 2008; Suarez et al. 2008; Suarez and Sessa 2009), suggesting that the angiogenic switch is delicately balanced by multiple families of microRNAs.

\footnotetext{
${ }^{6}$ These authors contributed equally to this work.

${ }^{7}$ Corresponding author

E-mail sharppa@mit.edu

Article published online ahead of print. Article and publication date are online at http://www.genesdev.org/cgi/doi/10.1101/gad.239681.114.
}

Here we report that global microRNA depletion breaks the balance of the angiogenic switch. MicroRNA-deficient tumors are highly hypoxic but poorly vascularized. The reduced angiogenic capacity in microRNA-deficient cancer cells is primarily mediated by derepression of FIH1 (factor inhibiting HIF-1 [hypoxia-inducible factor 1]), which inhibits HIF transcriptional activity.

\section{Results}

We set out to study tumor angiogenesis in a non-smallcell lung cancer (NSCLC) model driven by the KrasG12D oncogene accompanied by p53 loss (Kumar et al. 2009). Because the maturation of the vast majority of microRNAs requires Dicer1 (Bernstein et al. 2003; Gurtan et al. 2012), we generated Dicer1 knockout cancer cells to deplete the global microRNA population (Fig. 1A). Northern blot showed that mature microRNAs, such as miR-21a, let-7g, and miR-125b, are abundant in Dicer $1^{+/-}$cells but undetectable in Dicer $1^{-1-}$ cells (Fig. 1B). We performed small

(c) 2014 Chen et al. This article is distributed exclusively by Cold Spring Harbor Laboratory Press for the first six months after the full-issue publication date (see http://genesdev.cshlp.org/site/misc/terms.xhtml). After six months, it is available under a Creative Commons License (Attribution-NonCommercial 4.0 International), as described at http:// creativecommons.org/licenses/by-nc/4.0/. 


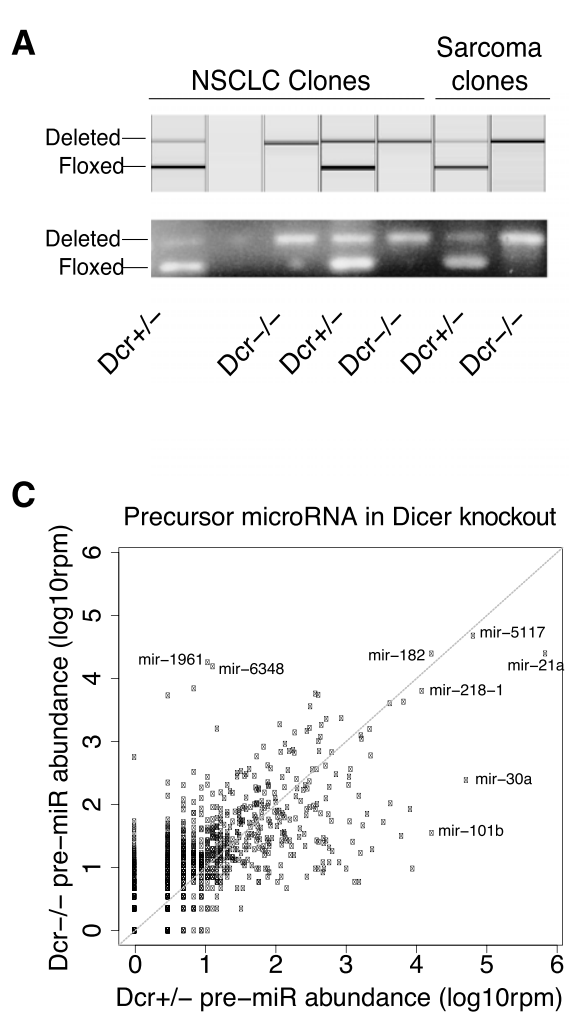

E

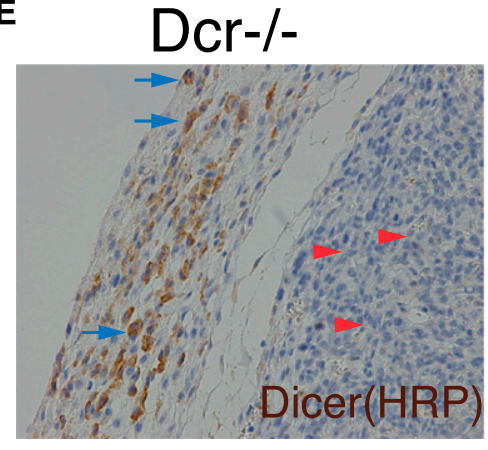

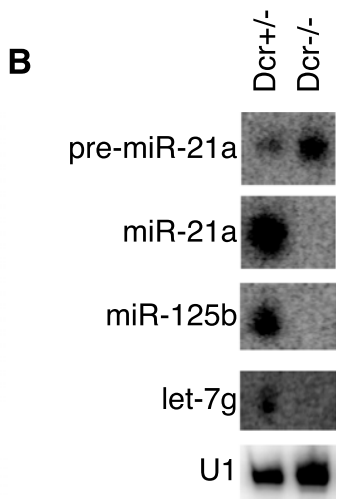

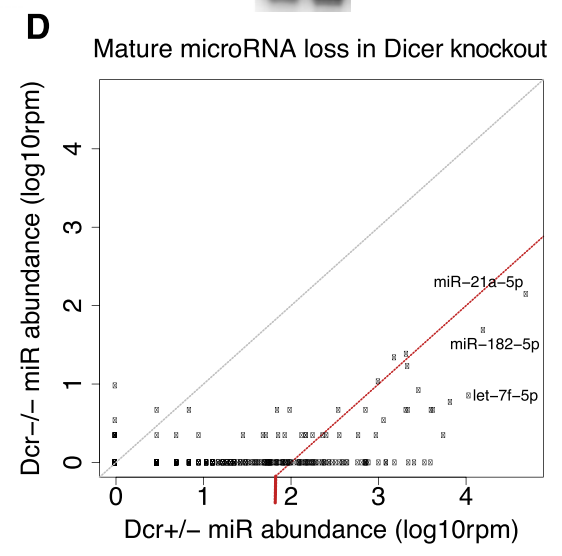

Dcr+/-

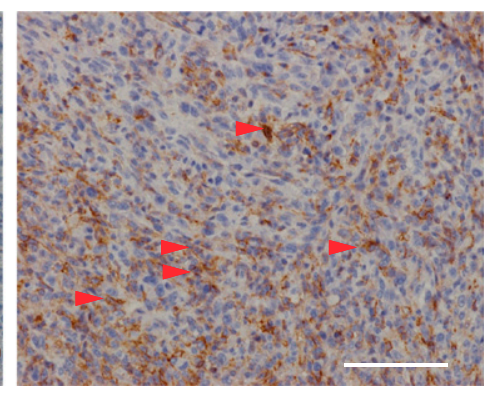

Figure 1. (A) Genotyping of Dicer1 heterozygous and knockout NSCLC clonal cell lines using capillary electrophoresis (top panel) and standard gel electrophoresis (bottom panel) showing loss of the wild-type (floxed) Dicer1 allele in Dicer1 knockout cells. Sarcoma clones with known Dicer1 genotypes were used as a reference. (Note that lane 2 is left blank.) (B) Northern blot of several representative microRNAs in Dicer ${ }^{+/-}$and knockout cells showing loss of mature microRNAs in Dicer1 knockout cells. (C) Scatter plot of pre-microRNA abundance from microRNA-seq in Dicer1 heterozygous and knockout cells. The gray dotted line represents the diagonal $(x=y)$. (D) Scatter plot of mature microRNA abundance from microRNA-seq in Dicer1 heterozygous and knockout cells showing global loss of mature microRNAs in Dicer1 knockout cells. The gray dotted line represents the diagonal $(x=y)$; the red dotted line represents the 100 -fold decrease $(x=y / 100)$. (E) Immunohistochemistry (IHC) of Dicer in sections of tumors induced by Dicer $1^{-/-}$ and Dicer ${ }^{+/-}$cells showing loss of Dicer protein staining in Dicer1 knockout tumors. Images were captured by a light microscope under $20 \times$ magnification. Bar, $100 \mu \mathrm{m}$. (HRP) Horseradish peroxidase. Red arrowheads indicate representative tumor cells. Blue arrows indicate representative peritumor host cells in Dicer $1^{-1-}$ tumors.
RNA sequencing (small RNA-seq) to capture microRNAs (thus also considered as microRNA-seq) and detected $>100$ mature microRNAs in Dicer ${ }^{+/-}$cells, with miR-21a-5p as the most abundant, followed by miR-182-5p and let-7 family members (Fig. 1C,D; Supplemental Table S2A). Dicer1 ${ }^{-/-}$ cells have a $>100$-fold decrease in mature microRNA levels compared with Dicer $1^{+/-}$cells, whereas the hairpin precursors (pre-microRNAs) are expressed at similar levels in both genotypes (Fig. 1C,D; Supplemental Table S2B). We therefore considered Dicer $1^{+/-}$and Dicer $1^{-/-}$cells as microRNAcompetent and microRNA-deficient cells, respectively.

Both Dicer $1^{+/-}$and Dicer1 $1^{-/-}$NSCLC cells induced tumors when injected into immunocompromised mice. Immunohistochemistry (IHC) showed that cancer cells stained positive for Dicer protein in tumors induced by Dicer $1^{+/-}$cells but not in those induced by Dicer $1^{-/-}$cells, whereas tumor-associated host tissue stained positive (Fig. 1E). We then performed analyses of tumor hypoxia and angiogenesis in Dicer $1^{+/-}$and Dicer $1^{-/-}$tumors. Hypoxyprobe (Raleigh et al. 1996; Varghese et al. 1976) staining showed that Dicer $1^{-/-}$tumors have higher levels of hypoxia than Dicer1 $1^{+-}$(Fig. 2A). Because hypoxic regions represent tissues with low oxygen levels, where normal cells activate a hypoxia-inducible response to provoke tumor angiogenesis (Carmeliet and Jain 2000; Weinberg 2007; Konisti et al. 2012), we expected to see more active angiogenesis with higher hypoxia. Surprisingly, immunofluorescence (IF) staining of an endothelial cell (EC)-specific marker, Isolectin B4, showed that Dicer $1^{-1-}$ tumors have significantly reduced tumorassociated vasculature (Fig. 2B). Quantitation of Isolectin B4 and Ki67 staining demonstrated that the proliferation of tumor-associated ECs was significantly decreased in Dicer1 knockouts (Fig. 2B). These data suggested that microRNA-deficient tumors have reduced angiogenesis despite being highly hypoxic. 

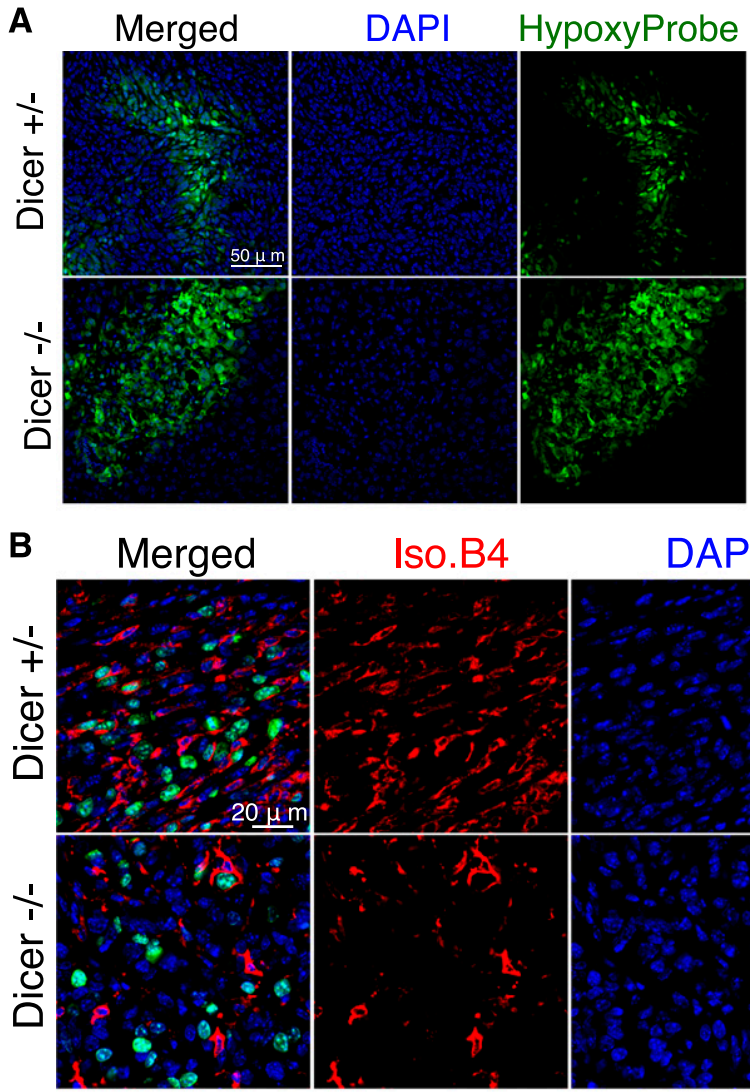

DAPI

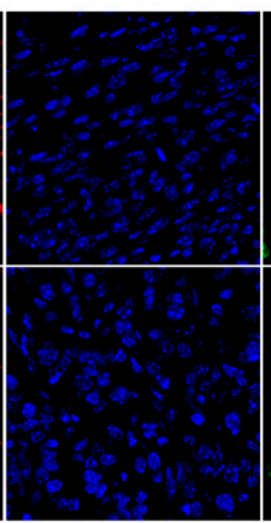

Tumor hypoxia

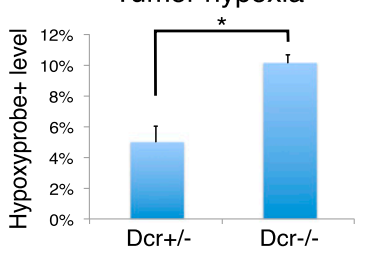

Figure 2. $(A)$ IF of sections of tumors induced by Dicer1 ${ }^{+/-}$and Dicer $1^{-/-}$cells showing increased level of hypoxia in Dicer $1^{-/-}$tumors. Channels are DAPI (blue) and Hypoxyprobe (green). Images were captured by a confocal microscope under $20 \times$ magnification. Bar, $50 \mu \mathrm{m}$. The right panel shows the quantification of tumor hypoxia level indicated by Hypoxyprobe staining sum intensity normalized by nuclei (DAPI). (B) IF of sections of tumors induced by Dicer $1^{+/}$and Dicer1 $1^{-/-}$cells showing decreased level of vasculature in Dicer1 $1^{-1-}$ tumors. Channels are DAPI (blue) and antibodies against Isolectin B4 (Iso.B4; red) and Ki67 (green). Images were captured by a confocal microscope under $40 \times$ magnification. Bar, $20 \mu \mathrm{m}$. The bottom left panel shows quantification of vasculature indicated by Isolectin $\mathrm{B}^{+}$cells (percentage). The bottom right panel shows quantification of the levels of EC proliferation indicated by Ki67 Isolectin $\mathrm{B}^{+}{ }^{+}$double-positive ECs normalized by total Isolectin $\mathrm{B} 4^{+}$ECs (percentage). Quantification was performed blind on three randomly chosen fields using CellProfiler. Data represent the average from three samples \pm standard error of the mean (SEM). The asterisk denotes statistical significance (t-test, $P<0.01)$.

\section{Endothelial cell density}

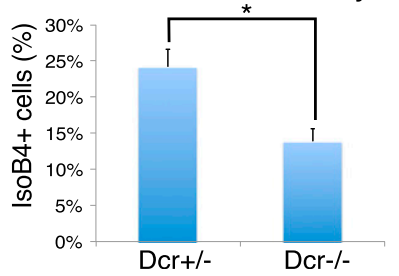

Endothelial cell proliferation

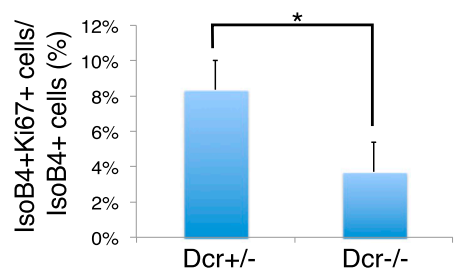

To understand the underlying gene regulation of tumor angiogenesis in microRNA-deficient cells, we profiled the transcriptomes of microRNA-competent and microRNA-deficient cells using messenger RNA sequencing (mRNA-seq) and identified populations of differentially expressed genes (Fig. 3A; Supplemental Table S1). The activity of microRNA families was analyzed from the degrees of derepression of their predicted target genes upon Dicer1 loss (Fig. 3B; Supplemental Fig. S1b,c). Many microRNA families exhibited strong silencing activity, as their target genes showed significant up-regulation upon microRNA loss (Fig. 3B; Supplemental Fig. S1b,c). Globally, of the 153 conserved microRNA families, 43 showed significant activity in these cells (Fig. 3B; Supplemental Table S3). The most active in Dicer $1^{+/-}$cells are miR-29, miR-202-3p, let-7/miR-98, miR-17, miR93/295, and miR-125 families (Fig. 3B). The relative activities of microRNAs on the predicted target mRNA transcriptome significantly correlate with their abundance in microRNA-seq data (Supplemental Fig. S1a), suggesting that depletion of the highly abundant microRNAs led to significant derepression of their targets.

Pathway analysis showed that mRNA genes downregulated upon microRNA depletion are highly enriched in angiogenesis functions, including gene sets with clustered functions such as angiogenesis, vasculature development, blood vessel morphogenesis, cardiovascular system development and function, cell migration, and migration of ECs (Fig. 3C; Supplemental Tables S4, S5). These genes significantly overlap with genes regulated by the $\alpha$ subunit of HIF-1 $\alpha$ (also known as HIF1A) (Fig. 3D). MicroRNAs generally act as repressors of their target genes (Bartel 2009); thus, genes down-regulated upon microRNA loss are likely due to indirect effects.

To investigate this indirect regulation of angiogenesis by microRNAs, we examined the expression of genes known to negatively regulate HIF. Among all known HIF antagonists, FIH1 (also known as Hif1an) is the most highly up-regulated in microRNA-deficient cells (Fig. 3E). Consistently, FIH1 protein level is also highly up-regulated 


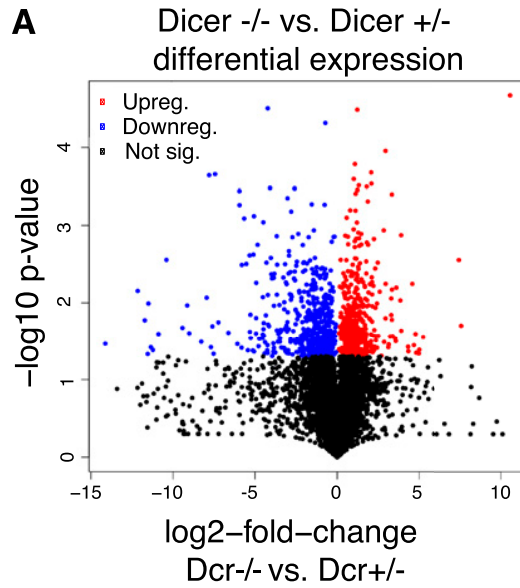

C

\begin{tabular}{lc}
\multicolumn{1}{|c|}{ GO analyses of Dicer -/- down genes } \\
Biological process & Adj. p-value \\
Translation & $2.05 \mathrm{E}-05$ \\
Blood vessel morphogenesis & $4.44 \mathrm{E}-05$ \\
Cell adhesion & $6.70 \mathrm{E}-05$ \\
Biological adhesion & $6.86 \mathrm{E}-05$ \\
Blood vessel development & $7.40 \mathrm{E}-05$ \\
Vasculature development & $1.04 \mathrm{E}-04$ \\
Regulation of cell proliferation & $1.47 \mathrm{E}-04$ \\
Angiogenesis & $2.47 \mathrm{E}-04$ \\
Embryonic limb morphogenesis & $3.11 \mathrm{E}-03$ \\
Embryonic appendage morphogenesis & $3.11 \mathrm{E}-03$ \\
Cell migration & $3.34 \mathrm{E}-03$
\end{tabular}

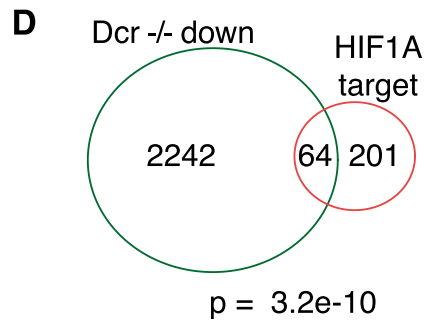

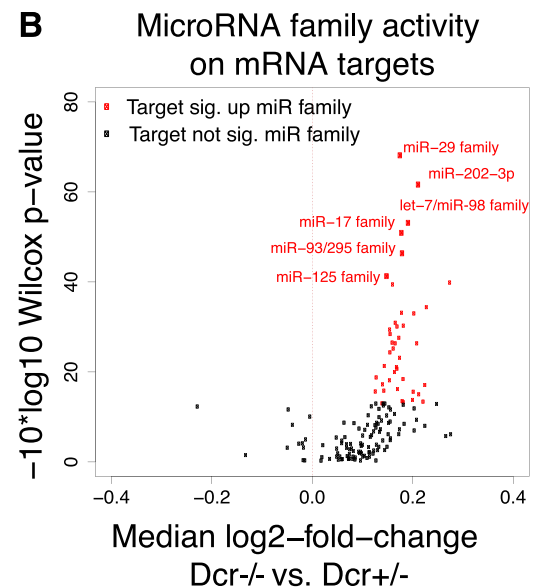

E

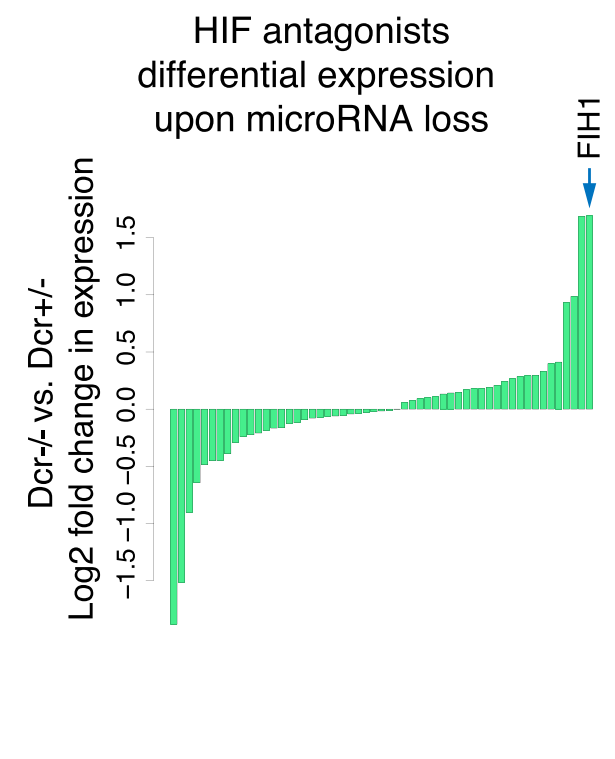

Figure 3. (A) Scatter plot of expressed coding mRNA genes in Dicer ${ }^{+/-}$and Dicer $1^{-/-}$NSCLC cells showing global differential expression between two genotypes. The $X$-axis is the $\log 2$ fold change of Dicer1 knockout over heterozygotes, and the $Y$-axis is the statistical significance $(-\log 10 P$-value). Genes significantly upregulated are shown as red dots. Genes significantly down-regulated are shown as blue dots. Genes not significantly changed are shown as black dots. (B) Global microRNA activity in the transcriptome of mRNAs in NSCLC cells depicted as a scatter plot of derepression of TargetScan-predicted conserved target gene sets of microRNAs grouped by TargetScan seed family. The $X$-axis is the median $\log 2$ fold change of microRNA target genes versus randomized control gene sets, and the $Y$ axis is the statistical significance $(-\log 10 P$ value). MicroRNA families with significant activity in target gene set repression are shown as red dots. $(C)$ Gene ontology (GO) analysis of genes significantly down-regulated in Dicer1 knockout showing enrichment in functional clusters of angiogenesis. (D) A Venn diagram of gene set overlap between genes significantly down-regulated in Dicer $1^{-1-}$ and target genes of HIFl $\alpha$ (HIF1A target). The target gene set of HIF1 $\alpha$ was retrieved from the Ingenuity Pathway Analysis (IPA) database. (E) A waterfall plot of differential expression of known HIF antagonist genes between Dicer1 knockout and heterozygotes showing that the top upregulated gene is FIH1. upon microRNA depletion (Figs. 4B, 5A,C). FIH1 hydroxylates an asparagine residue of HIF- $1 \alpha$ in its C-terminal transactivation domain (McNeill et al. 2002; Koivunen et al. 2004). This hydroxylation blocks the association of HIF with the transcriptional coactivators $\mathrm{CBP} / \mathrm{p} 300$ and thus inhibits transcriptional activation (Mahon et al. 2001; Lando et al. 2002). FIH1 is a microRNA target with an annotated 5116-base-pair (bp) 3' untranslated region (UTR) predicted to harbor 473 microRNA-binding sites, including five let-7 sites and four miR-125 sites (Supplemental Fig. S1d). These results led us to hypothesize that FIH1 derepression might be responsible for the lower level of HIF transcriptional activity upon loss of microRNAs.

To test the role of FIH1 derepression, we generated FIH1 knockout and FIH1;Dicer1 double-knockout NSCLC cells. We adopted a recently developed genome-editing technology based on the RNA-guided nuclease Cas9 from the microbial CRISPR adaptive immune system (Fig. 4A; Cong et al. 2013; Mali et al. 2013). Using an algorithm that minimizes predicted genomic off-target sites (Hsu et al.
2013), we designed a single-guide RNA (sgRNA) targeting a genomic region almost immediately after the start codon of FIH1 on chromosome 19 (Fig. 4A). We transfected microRNA-deficient cells with a construct coexpressing the human codon-optimized Streptococcus pyogenes Cas 9 and the FIH1 targeting sgRNA. Following incubation to allow genome editing, single cells were isolated by fluorescence-activated cell sorting (FACS) using flow cytometry into 96-well plates. Individual colonies were isolated and expanded to establish clonal cell lines. FIH1 protein level was determined by Western blot, and seven out of 12 (58\%) of the clones completely lost FIH1 protein (Fig. 4B; Supplemental Fig. S8), suggesting that these clones are FIH1-null mutants.

We genotyped the clonal FIH1-null mutants by PCRamplifying the targeted FIH1 locus followed by Illumina sequencing (Miseq). This revealed multiple FIH1-disrupting mutations, most of which are small deletions causing frameshifts (Fig. 4C; Supplemental Fig. S2). Clonal cell lines showed either one or two isoforms of the FIH1 alleles, 
Chen et al.

A

Mouse FIH1 locus (chr. 19)

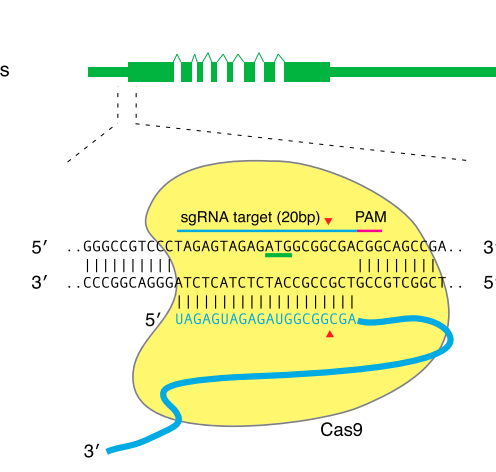

B $\frac{1}{\frac{1}{4}}$

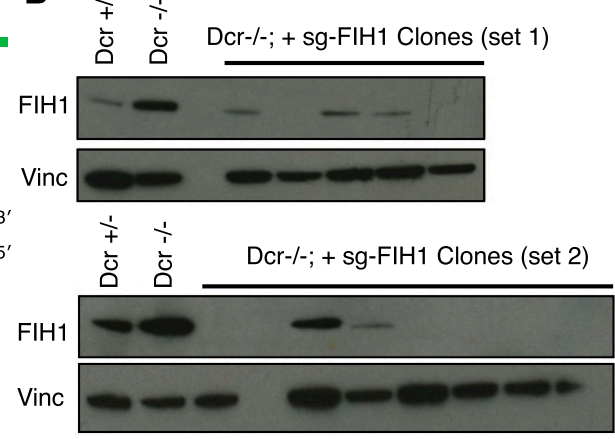

C

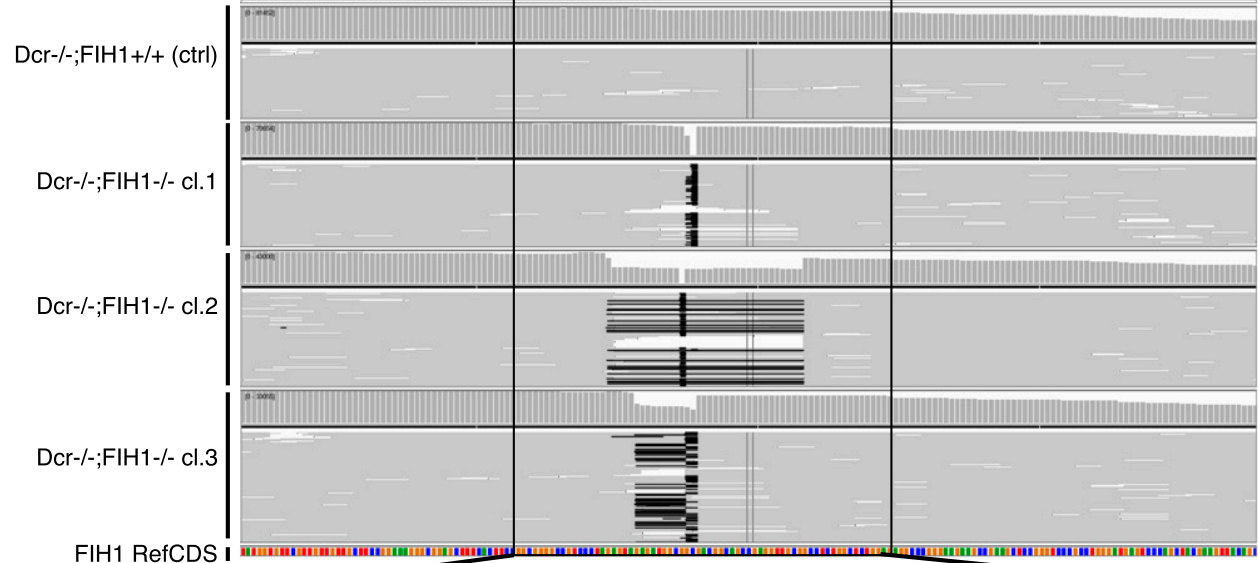

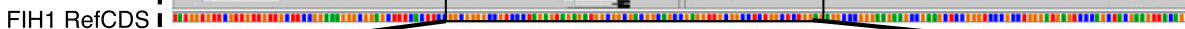

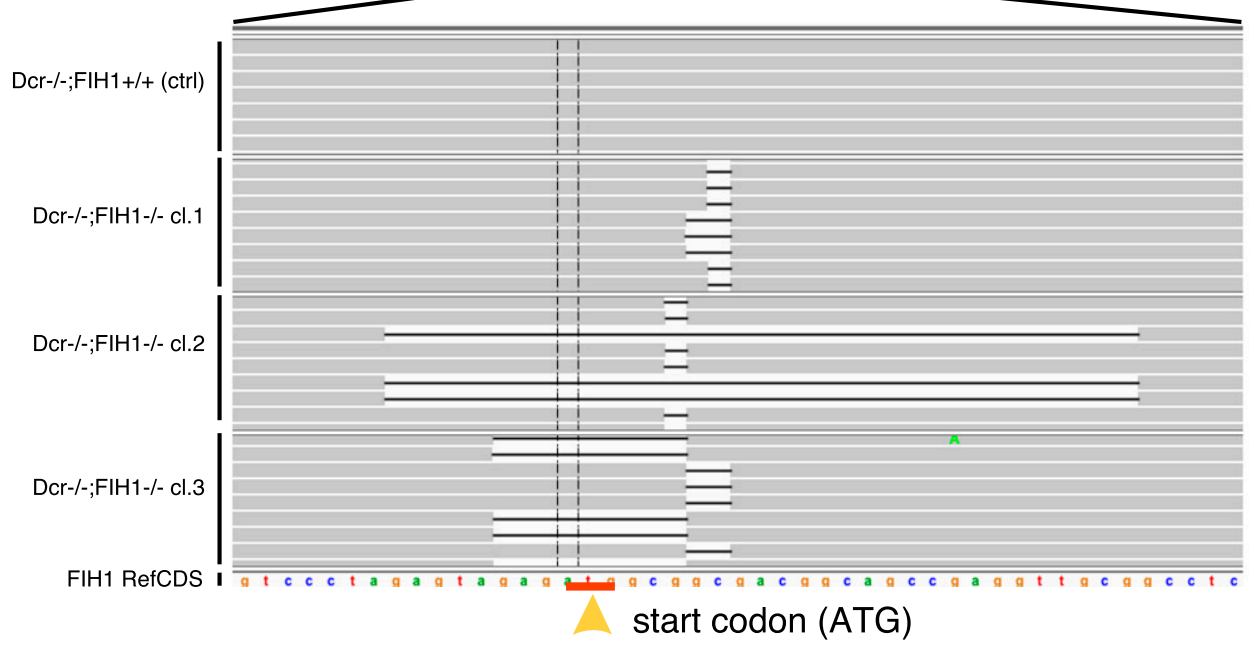

Figure 4. (A) Schematic representation of FIH1 targeting using the CRISPR/Cas9 system. (Top panel) Gene structure model of FIH1 in mouse chromosome 19 (not drawn to scale). (Bottom panel) sgRNA sequence and targeted region sequence. The start codon (ATG) and the PAM (CGG) are indicated. (B) Western blot of FIH protein from clonal cell lines after sg-FIH1 and Cas9 transfection showing loss of FIH1 protein expression in seven out of 12 cell lines. $(C)$ Miseq of the FIH1 exon1 genomic PCRs of clonal cell lines in a Dicer1 ${ }^{-/-}$ background.

suggesting that these are either homozygous or transheterozygous FIH1-null cells, respectively (Fig. 4C; Supplemental Figs. S2, S3). We also amplified and sequenced the top two predicted off-target loci, Evc2 and Fam126, in these cell lines. Neither locus showed any insertion or deletion, suggesting minimal off-target activity (Supplemental Fig. S4). The FIH1 knockout cell lines in the
Dicer $1^{+/-}$and Dicer1 $1^{-/-}$background are termed FIH1 knockout $\left(\right.$ Dicer1 $\left.^{+/-} ; \mathrm{FIH1}^{-/-}\right)$and Dicer1;FIH1 double knockout (Dicer1 $\left.{ }^{-/-} ; \mathrm{FIH1}^{-/-}\right)$.

We then investigated the roles of microRNA loss and FIH1 in HIF transcriptional activity. Using a HIFresponsive element (HRE) reporter assay, we found that microRNA-deficient cells have significantly lower levels 
A

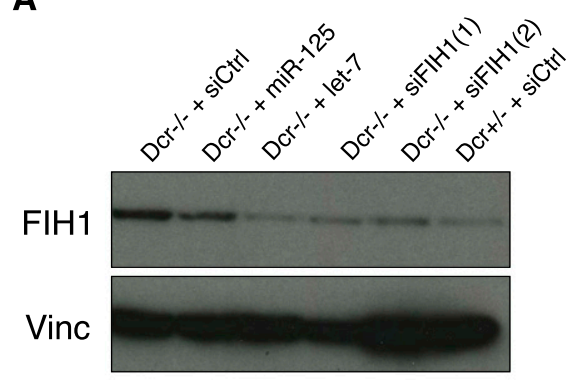

C

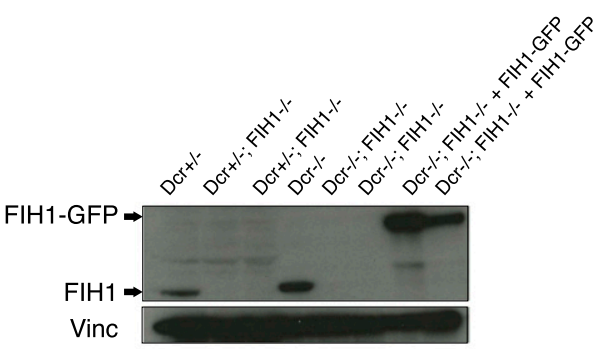

B FIH1 knockdown

D

FIH1 CRISPR knockout and cDNA Rescue
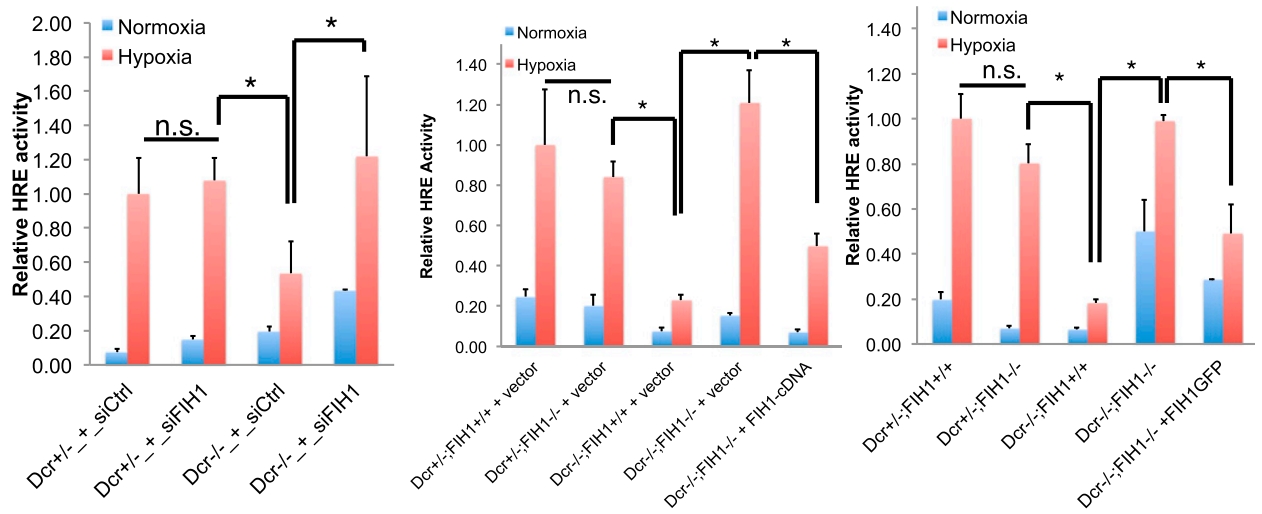

E

$\mathbf{F}$

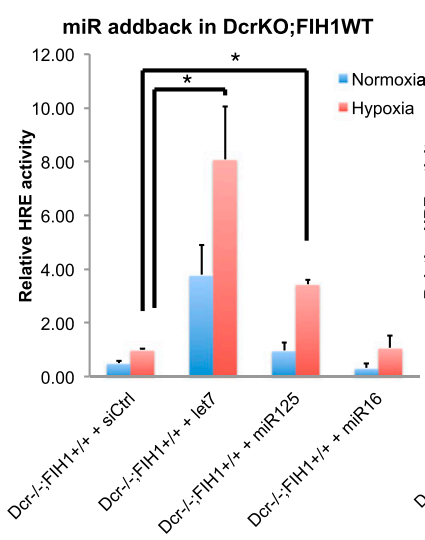

miR addback in DcrKo; FIH1KO

1.60
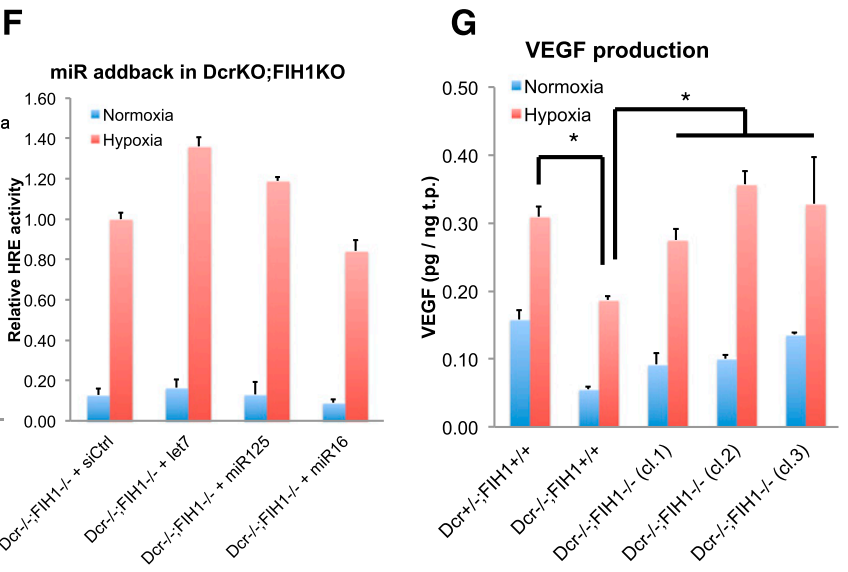

Figure 5. (A) Western blot of FIH1 protein following siRNA or microRNA transfection showing transfection of FIH1 targeting siRNAor microRNA-reduced FIH1 protein in Dicer1 knockout cells to a level similar to Dicer1 heterozygous. $(B)$ HRE reporter assay of Dicer $1^{+/-}$and Dicer $1^{-/-}$cells showing that Dicer1 knockout cells reduced HRE activity compared with Dicer1 heterozygotes and that FIH1 knockdown increased HRE activity only in a Dicer1 knockout background. (C) Western blot of FIH1 protein with FIH1 knockout and rescue showing loss of FIH1 protein in CRISPR/Cas9-generated FIH1 knockout cell lines and re-expression after transfection of a plasmid with human FIH1 cDNA. (D) HRE assay of FIH1 knockout and rescue showing increase of HIF transcription activity upon FIH1 knockout only in Dicer1 knockout background, and suppression of HRE by re-expressing FIH1. (E) HRE assay of microRNA addback in Dicer1 knockout FIH1 wild-type cells showing significant increase of HIF transcription activity upon transfection of FIH1 targeting microRNAs (let-7 and miR-125). (F) HRE assay of microRNA addback in Dicer1;FIH1 double-knockout cells showing diminished effects of microRNA addback on HIF transcription activity. For HRE assays in $B$ and $D-F$, the raw measurements were normalized to the value of control group hypoxia conditions. $(G)$ VEGF ELISA assay showing reduced VEGF production in Dicer1 knockout cells compared with Dicer1 heterozygotes. VEGF production increased upon FIH1 knockout in the Dicer1 knockout background to a level similar to Dicer $1^{+/-} ; F I H 1^{+/+}$. (pg/ng t.p) Picograms of VEGF per nanogram of total protein. Data represent the average from three samples \pm SEM. The asterisk denotes statistical significance $(t$-test, $P<0.01)$. (n.s.) Not significant.

of HIF transcriptional activity as compared with heterozygotes (Fig. 5B). Knocking down FIH1 using siRNAs reduced FIH1 protein by $\sim 80 \%$ (Fig. $5 \mathrm{~A}$ ), leading to increased HIF transcription activity in microRNA-deficient cells but with little effect in microRNA-competent cells (Fig. 5B). Complete FIH1 knockout led to loss of FIH1 
protein, which was rescued by transfection of a plasmid carrying a human FIH1 coding region transgene (Fig. 5C). Knocking out FIH1 in the microRNA-competent background did not alter HIF transcriptional activity, whereas knocking out FIH1 in the microRNA-deficient background increased HIF transcriptional activity back to a level comparable with that of the parental microRNAcompetent, FIH1 wild-type cells $\left(\right.$ Dicer $1^{+/-}$; FIH1 $\left.^{+/+}\right)($Fig. 5D). Re-expression of human FIH1 protein in Dicer1;FIH1 double-mutant cells suppressed HIF transcriptional activity (Fig. 5D).

As direct evidence that the up-regulation of FIH upon Dicer loss was due to loss of microRNAs, reintroducing the FIH1 targeting microRNAs let-7 and miR-125 into Dicer1 ${ }^{-/-} ; \mathrm{FIH1}^{+/+}$cells decreased FIH1 protein level (Fig. 5A) and augmented HIF transcriptional activity (Fig. 5E), whereas transfection of miR-16, which does not target FIH1, did not change HIF transcriptional activity (Fig. 5E). In Dicer1;FIH1 double-knockout cells, reintroduction of let-7 or miR-125 had an insignificant effect compared with control siRNA (Fig. 5F). These data suggest that FIH1 is epistatic to Dicer1; i.e., it acts downstream from Dicerdependent microRNAs in HIF transcriptional activity.

VEGF is a major direct target of HIF and is a key regulator of angiogenesis (Leung et al. 1989; Carmeliet and Jain 2000). We used ELISA to quantitatively measure the level of VEGF. MicroRNA-deficient cells produced less VEGF as compared with their parental microRNAcompetent cells (Fig. 5G). Dicer1;FIH1 double-knockout cells produced more VEGF than their FIH1 wild-type counterparts, at a level close to Dicer1 ${ }^{+/-}$(Fig. 5G). These data suggest that FIH1 is also downstream from Dicer1 in VEGF production.

Dicer1;FIH1 double-knockout cells were then assayed for tumor hypoxia and angiogenesis in vivo. We subcutaneously injected Dicer $1^{+/-}$, Dicer1 ${ }^{-/-}$, and Dicer $1^{-/-} ; \mathrm{FIH}^{-/-}$ NSCLC cells into the flanks of nude mice and then harvested tumor samples. The Dicer1;FIH1 double-knockout tumors were significantly less hypoxic as compared with the parental Dicer1 knockout, similar to that of Dicer $1^{+/-}$ (Fig. 6A,B). This result was confirmed with HypoxyProbe IHC (Supplemental Fig. S5a,c). We then analyzed tumor vasculature using Isolectin B4 IF. Dicer1;FIH1 doubleknockout tumors had significantly increased vasculature density compared with the Dicer1 single knockout, at a level similar to Dicer $1^{+/-}$(Fig. 6A,C). This result was confirmed with IHC using an independent EC marker, CD31 (Supplemental Fig. S5b,d). These data revealed that FIH1 knockout reversed the defects of microRNA loss in tumor hypoxia and angiogenesis, suggesting that FIH1 is the major target downstream from microRNAs for these phenotypes in vivo.

Because the primary effect of microRNA repression is mediated by the 3' UTR (Bartel 2009), we set out to test direct repression by microRNAs specifically on the FIH1 locus. We mutagenized the FIH1 3' UTR by CRISPR/Cas9 using a combination of multiple sgRNAs targeting the FIH1 3' UTR (Supplemental Fig. S6a). We FACS-sorted single cells after mutagenesis and isolated multiple clonal cell lines. We PCR-amplified the genomic region of the

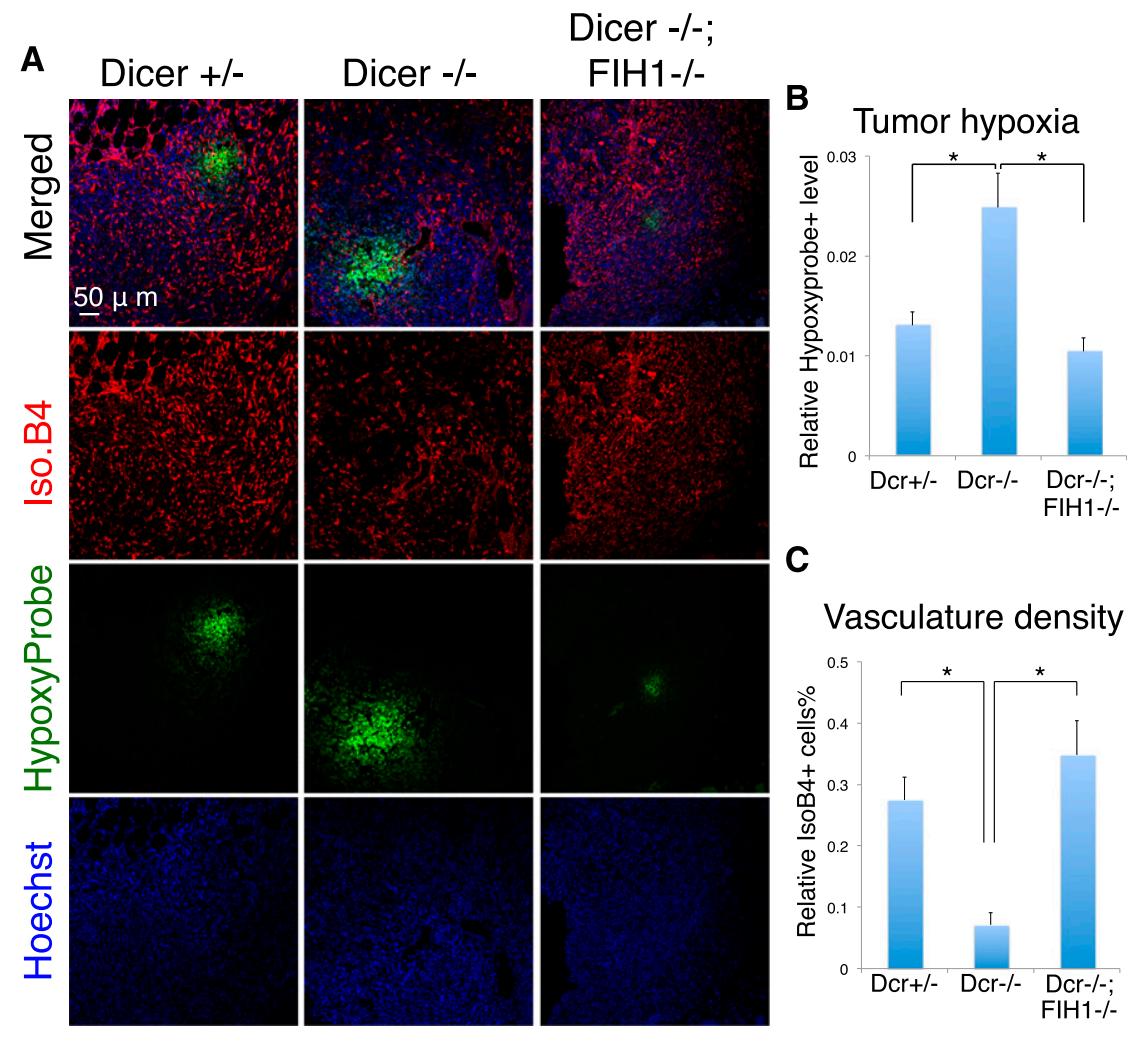

Figure 6. (A) IF of sections of tumors induced by Dicer1 heterozygous, Dicer1 knockout, and Dicer1;FIH1 double-knockout cells. Channels are Hoechst (blue), Hypoxyprobe (green), and Isolectin B4 (Iso.B4, red). Images were captured by a confocal microscope under $20 \times$ magnification with $0.5 \times$ digital zoom. Bar, $50 \mu \mathrm{m}$. (B) Quantification of the levels of hypoxia intensity indicated by relative Hypoxyprobe staining intensity. Quantification was performed blind on three randomly chosen fields using CellProfiler. (C) Quantification of vasculature density indicated by relative Isolectin $\mathrm{B} 4^{+}$cells (percentage). Quantification was performed blind on three randomly chosen fields using CellProfiler. Data represent mean \pm SEM. The asterisk denotes statistical significance (t-test, $P<0.01$ ). 
FIH1 3' UTR of these clonal cell lines, which revealed various monoallelic or biallelic deletions in the FIH1 3' UTR (Supplemental Fig. S6b). These FIH1-3' UTR mutants showed elevated levels of $\mathrm{FIH} 1$ protein as a result of partial loss of microRNA repression (Supplemental Fig. S6c). Among these mutants, we focused on the cell line that has the largest deletion in the FIH1 3' UTR. We performed Illumina sequencing and Sanger sequencing for this clone. Sequencing revealed that it harbors a homozygous 3770-bp deletion, which deletes the majority of the microRNAbinding sites in the $3^{\prime}$ UTR (Fig. 7A,B). This cell line showed a high level of protein up-regulation compared with FIH1 3' UTR wild type (Supplemental Fig. S6c, mutant 4). We performed the HRE assay and found that this mutant showed down-regulation of HIF transcriptional activity to a level comparable with microRNA-deficient cells (Fig. 7C). These data strongly indicate that microRNAs directly repress FIH1 to regulate HIF transcriptional activity.

\section{A}

FIH 3'UTR mutant

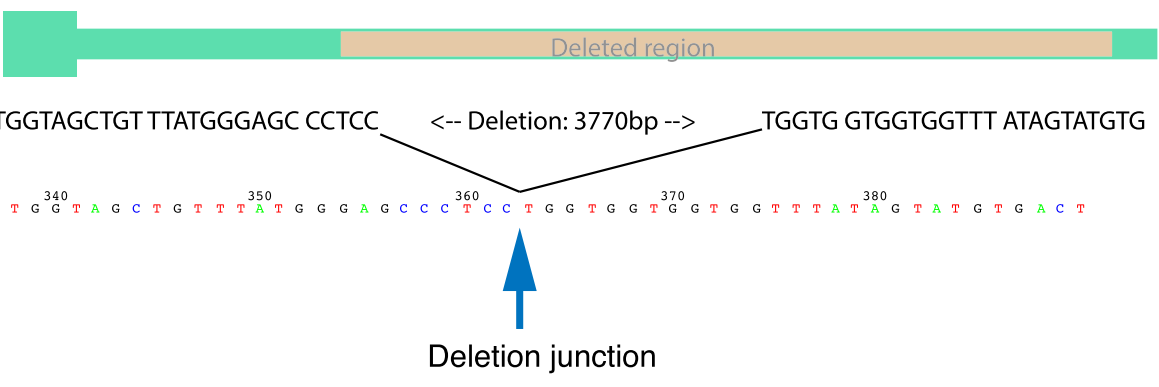

B
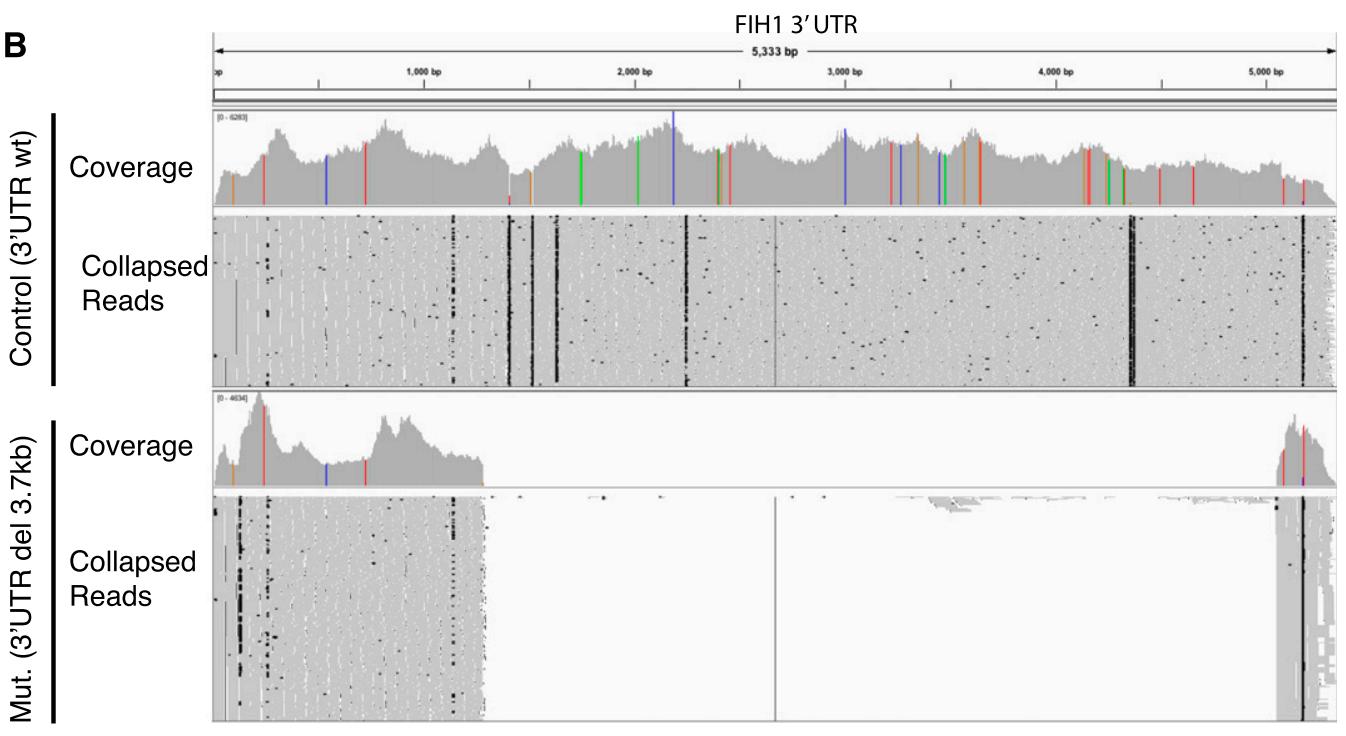

C

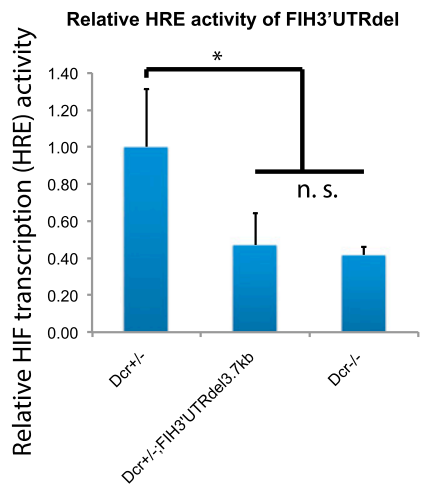

Figure 7. (A) Sanger sequencing of genomic PCR of a clonal FIH1 3' UTR mutant cell line harboring a homozygous 3.7-kb deletion generated using CRISPR/Cas9. (B) Captured Illumina sequencing of the FIH1 3' UTR of the mutant cell line harboring a homozygous 3.7-kb deletion. $(C)$ HRE assay of representative FIH1 3' UTR mutant cell lines showing repressed HIF transcription activity. Data represent mean \pm SEM. The asterisk denotes statistical significance $(t$-test, $P<0.01)$. (n.s.) Not significant. 
We tested the tumor growth rate of Dicer $1^{+/-}$, Dicer $1^{-/-}$, Dicer $1^{-/-}$;FIH1 $1^{-/-}$, and Dicer $1^{+/-}$;FIH1-3' UTR-mutant cell lines. The FIH1 3' UTR mutant, which derepresses FIH1, grows more slowly than its parental cell line, Dicer $^{+/-}$, at a rate similar to Dicer $1^{-/-}$(Supplemental Fig. S7). These data suggest that derepression of FIH1 by loss of either microRNAs or microRNA-binding sites suppresses tumor growth. The Dicer1 $1^{-/-} ; F_{H H} 1^{-/-}$double mutant grows more slowly than Dicer $1^{-/}$(Supplemental Fig. S7), implying that loss of FIH1 in this context may have effects other than angiogenesis that affect tumor growth. In general, tumor hypoxia, angiogenesis, and HIF signaling are delicately controlled by multiple components and may not linearly translate into tumor growth (Semenza 2003; Schofield and Ratcliffe 2004; Kaelin 2008; Kaelin and Ratcliffe 2008).

We went on to test the dependence of regulation by FIH1 on the asparagine residue at the C-terminal domain of HIF1A. We generated a HIF1A-N803A mutant construct and reintroduced it into the Dicer $1^{-/-}$cells by transfection. HIF1A-N803A transfection leads to a significantly higher increase of HRE activity as compared with HIF1A wild type in the presence of high levels of FIH1 (Supplemental Fig. S9). These data and the previous results suggest that $\mathrm{FIH} 1$ is actively repressing the wild-type HIF1A through hydroxylation of N803 in these NSCLC cells.

FIH1 has been recently shown to have multiple targets (Zheng et al. 2008; Janke et al. 2013); in particular, FIH1 has been shown to target NOTCH for transactivation in other cellular systems (Zheng et al. 2008; Wilkins et al. 2009). In RNA-seq data from the NSCLC cell lines, the vast majority (36 out of 38) of expressed NOTCH target genes are not significantly altered upon FIH1 derepression following loss of microRNA regulation (Supplemental Fig. S10; Supplemental Table S6). These data suggest that NOTCH is not a major target of FIH1 in these cell lines.

A previous study (Dayan et al. 2006) documented that CA9 (Car9 in mice) and PHD3 (Egln3 in mice) are sensitive to FIH1. We analyzed the expression of these genes in our RNA-seq data set and found that Car9 and Egln3 are downregulated as FIH1 is derepressed upon microRNA loss in Dicer $1^{-/-}$cells. In the list of FIH1-sensitive and FIH1insensitive genes in the study by Dayan et al. (2006), we found that the FIH1-sensitive genes are mostly (12 out of $14,86 \%$ ) down-regulated in Dicer $1^{-/-}$cells (compared with random, $\chi^{2}$ test, $\left.P=0.02\right)$, and FIH1-insensitive genes behave randomly $\left(\chi^{2}\right.$ test, $P=0.61$ ) (Supplemental Table S7). These data suggest that FIH1-sensitive genes are modulated by FIH1 and microRNAs.

\section{Discussion}

Mutations and misregulation of Dicer1 have been implicated in tumorigenesis in mouse models of NSCLC (Kumar et al. 2007, 2009), sarcoma (Ravi et al. 2012), and retinoblastoma (Nittner et al. 2012) as well as various human cancers (Kumar et al. 2009; Heravi-Moussavi et al. 2012). One of the first indications that microRNAs control malignant properties of human cancers was the strong correlation between low levels of let-7 microRNAs and progression of NSCLCs (Takamizawa et al. 2004; Lu et al. 2005). Mouse models of this disease recapitulated this correlation (Kumar et al. 2007, 2008). Thus, it was unexpected to find that Dicer-null NSCLC cells are defective for angiogenesis due to an inability to respond to hypoxia. This highlights the complexity of regulation of systems of genes by microRNAs in relationship to cancer. For example, let-7 microRNA can act as a tumor suppressor by suppressing growth rates through repression of the RAS pathway and sets of oncofetal genes (Gurtan et al. 2013), while at the same time, let-7 has oncogenic activity by promoting angiogenesis by repressing the activity of FIH1. Let-7 and miR-125 suppress FIH1, which suppresses the transcriptional activity of HIF and thus angiogenesis in response to hypoxia. In fact, $\mathrm{FIH} 1$ is highly responsive to changes in microRNA levels, with an extensive 3' UTR with hundreds of target sites for multiple microRNA families. Furthermore, we show that deletions in the 3' UTR of FIH1 increase its expression and accordingly decrease the activity of HIF.

Several microRNA families generated by Dicer are known to be required for blood vessel formation during mouse embryonic development (Yang et al. 2005; Suarez et al. 2008). However, the delicate balance of the angiogenic switch is highly context-dependent /Carmeliet and Jain 2000), and the pleiotropic regulatory functions of microRNAs vary from cell type to cell type. Several microRNAs have been shown to regulate hypoxia response and angiogenesis in different cancer cell lines, such as miR-9 (Zhuang et al. 2012), miR-210 (Kelly et al. 2011), let-7 (Chen et al. 2013), and miR-29a (Wang et al. 2013). These microRNAs are thought to function in either a hypoxia-dependent manner via HIF (Kelly et al. 2011; Chen et al. 2013) or a hypoxia-independent manner via other pathways, such as TGF- $\beta$, JAK/STAT (Zhuang et al. 2012; Wang et al. 2013), or EGFR signaling (Shen et al. 2013).

Many microRNAs are predicted to actively repress FIH1, which hydroxylates the asparagine residue of HIF$1 \alpha$ at its transactivation domain, thus repressing HIF transcription activity (Mahon et al. 2001; McNeill et al. 2002). Tumor cells respond to hypoxia by activating the HIF pathway, which turns on the production of VEGF and other adaptive response genes (Carmeliet and Jain 2000). In a head and neck carcinoma model, it was shown that miR-31 suppresses FIH to activate the HIF pathway (Liu et al. 2010). In the absence of Dicer1 and thus virtually all microRNAs, FIH1 is highly derepressed and inhibits the normal function of HIF-1 $\alpha$, thereby suppressing the response to hypoxia. This interference caused reduced tumor angiogenesis in Dicer1-null tumors even though the tumor hypoxia level is high. Using RNA-guided genome engineering technology based on CRISPR/Cas9, we showed that loss-of-function mutations in FIH1 reversed the defect of Dicer1-null cells in hypoxia response and tumor angiogenesis. Furthermore, deletion of several kilobases of the 3' UTR region in the FIH1 gene, which abolishes microRNA regulation and leads to derepressed FIH protein, 
suppresses HIF signaling. FIH1 is repressed in the presence of normal microRNAs (Dicer $1^{+/-}$cells) and thus is expressed at a low level. FIH1 knockout in Dicer $1^{+/-}$cells has no effect in the HRE because the basal expression level is low, consistent with the fact that FIH1 knockout mice show no dysregulation of HIF1 (Zhang et al. 2010). Only upon loss of microRNA repression by loss of either all microRNAs through Dicer1 deletion or microRNAbinding sites through $3^{\prime}$ UTR deletion is the effect of FIH1 on the HRE manifested.

We observed a global regulation of microRNAs on FIH1 in a panel of human cancer cell lines, with strong correlation in solid tumor types and no correlation with hematopoietic or lymphatic malignancies (data not shown). This may be explained by the fact that angiogenesis and hypoxia are more prominent in solid tumors compared with hematopoietic malignancies. Thus, microRNA regulation of FIH1 is general across many types of human cancers. The Dicer-microRNA-FIH1-HIF pathway may have important roles in tumor hypoxia response and angiogenesis in human cancer.

\section{Materials and methods}

\section{CRISPR-mediated genome editing of the FIH1 coding region}

sgRNAs targeting FIH1 were designed using tools described at http://tools.genome-engineering.org (Cong et al. 2013; Hsu et al. 2013). The guided sequence with minimal off-target gene targeting and high on-target score was chosen $\left(5^{\prime}\right.$-TAGAGTAGAG ATGGCGGCGA-3'). The oligo pair (mFIH-ex1-g-F1 and mFIHex1-g-R1) was annealed and cloned into BbsI-digested vector pX264Long to generate a construct, sg-FIH-ex1-F1-pX264Long. This construct was transfected to Dicer1 $1^{+/}$and Dicer1 $1^{-/-}$ NSCLC cells. Plasmid transfection was performed using Lipofectamine 2000 (Life Technologies). After 48 or 72 h, cells were cloned using FACS, and colonies were expanded for genotyping, RNA analysis, and protein analysis. Mutations were genotyped by genomic PCR followed by sequencing.

The oligos to generate the guide sequence for FIH1 targeting were mFIH-ex1-g-F1 (5'-CACCGTAGAGTAGAGATGGCGGC GA-3') and mFIH-ex1-g-R1 (5'-AAACTCGCCGCCATCTCTA CTCTAC-3').

The primers used for FIH1 genotyping and sequencing were mFIH-F1 (GGGCCGTCCCTAGAGTAGAG), mFIH-R3 (GCGT TTCCCCTGCTGTTTATTGAT), mFih_1kbfltss 1 F (CTAAGC GAGTCGGCCTTATG), mFih_1kbfltss 2 F (CTAAGCGAGTC GGCCTTATG), mFih_1kbfltss 3 F (ATTTCGTGGGCTTGTTT GTC), mFih_1kbfltss 4 F (GTGGACAGAGGCTTGAGAGG), mFih_1kbfltss 5 F (GCAATATTTCGTGGGCTTGT), mFih_ 1 kbfltss 6 F (CTAAGCGAGTCGGCCTTATG), mFih_1kbfltss 7 F (ATGACAATCTTGGCCTCCTG), mFih_1kbfltss 8 F (GTG GACAGAGGCTTGAGAGG), mFih_1kbfltss 9 F (ATGACAATC TTGGCCTCCTG), mFih_1kbfltss $10 \mathrm{~F}$ (ATTTCGTGGGCTTGT TTGTC), mFih_1kbfltss 1 R (CCTCTCAAGCCTCTGTCCAC), mFih_lkbfltss 2 R (TCCACCACACCTTCAAACAA), mFih_ $1 \mathrm{kbfltss} 3 \mathrm{R}$ (CCTCTCAAGCCTCTGTCCAC), mFih_1kbfltss $4 \mathrm{R}$ (TCCACCACACCTTCAAACAA), mFih_1kbfltss 5 R (CCTC TCAAGCCTCTGTCCAC), mFih_1kbfltss 6 R (GGATGCCCTG GTTCTACTGA), mFih_lkbfltss 7 R (CATAAGGCCGACTCG CTTAG), mFih_lkbfltss 8 R (GGATGCCCTGGTTCTACTGA), mFih_lkbfltss 9 R (CCTCTCAAGCCTCTGTCCAC), and mFih_ 1kbfltss 10 R (GGATGCCCTGGTTCTACTGA).
Primers for off-target genotyping and sequencing were Fam126a_Fihexlf1OT $4 \mathrm{~F}$ (AGCAATGTGCAAATGTGGTC), Fam126a_Fihex1f1OT 4 R (GCAGAACCTACCAGCAGAGG), Evc2_Fihex1f1OT 4 F (TGCTGAGATGGTATCGCTTG), and Evc2_Fihex1f1OT 4 R (CTTCGCTACAGCATGGAGGT).

\section{CRISPR-mediated genome editing of the FIH1 3' UTR}

sgRNAs targeting the FIH1 3' UTR were designed similarly. The following guides were used simultaneously to generate deletions in the FIH1 3' UTR: sg01-4_plus (GTATTGCACGCTGCACTTAA), sg01-12_plus (GACTCCACTCCCATTTGGAA), sg01-17_minus (GGTGAGAAACCTTTCCAAAT), sg06-2_plus (GTTTATGGG AGCCCTCCTCG), sg06-14_plus (GTATCTGTTAAGAGGG AATG), sg14-2_plus (GTTGCGCCCCACCTGTGACA), sg143_plus (TCGAAGCACTTGAGCTTGTG), sg14-6_plus (GAGCC TAGGTATGTGCAAGG), sg14-16_plus (TGGTCCAGCACAGG CTGTCT), sg14-4_minus (ACCСCTCCTTGCACATACCT), sg14-11_minus (GACAGATCCCTGTCACAGGT), and sg214_plus (CACTTAATAAACGGCTGTGG).

Genomic DNAs were extracted from clonal cell lines. Genomic $\mathrm{PCR}$, genotyping, and sequencing were performed as described above using the following primers: mFih-F6 (TGCTTCGTTGAT GAGGACAGGACA), mFih-R14 (AATTTAGAAGGGAGTGGC GACAGG), mFih-F7 (GCAGTACAGCGTGAACCCCAGATA), and mFih-R13 (TAAACCACCACCACCACAGCC).

\section{Genomic PCR Miseq}

Genomic PCR products were directly purified using DCC kit or gel-purified using a gel extraction kit (Zymo). Barcoded DNA libraries were prepared using the Nextera XT kit (Illumina). Normalized libraries were subjected to Miseq following the manufacturer's protocols.

\section{Miseq data analysis}

Miseq reads were mapped to amplicons with bwa paired-end mapping using a custom bwa index. Indels were called using VarScan (Koboldt et al. 2012) and BreakDancer (Chen et al. 2009) with manual validation using IGV (http://www.broadinstitute. org/software/igv).

\section{Mice and cell lines}

NSCLC cell lines were derived using a previously described mouse model (Kumar et al. 2009). Tumors were induced in the lung by intratracheal injection of Ad-Cre virus to activate $K$-ras and delete p53 and Dicer1 (Kumar et al. 2009). Cell lines were established from lung tumors with genotype KRas-G12D, $\mathrm{p} 53^{-/-}$, Dicer $1^{f /}$. Enforced Dicer1 homozygous deletion was induced in vitro by administration of adenovirus encoding Cre-GFP and FACS sorting of $\mathrm{GFP}^{+}$single cells or by infection with retrovirus encoding ER-Cre, treatment with 4-OH-tamoxifen, and FACSsorting of single cells. Multiple paired single-cell clonal lines were derived. Clonal lines were genotyped for Kras and $p 53$ as described previously (DuPage et al. 2009) and genotyped for Dicer1 using the following primers: MC113 (5'-AGCATGGGGGCACCCTGGT CCTGG-3'), MC93 (5'-CATGACTCTTCAACTCAAACT-3'), and MC94 (5'-CCTGACAGTGACGGTCCAAAG-3').

Murine sarcoma cell lines were derived by Ravi et al. (2012) and used as a genotyping control. Cells were cultured in standard DMEM $+10 \%$ FBS unless otherwise specified. Isogenic Dicer1 heterozygotes and Dicer1 knockout lines were infected with retrovirus encoding Luciferase-GFP, and stable clonal cell lines were established from FACS-sorting of $\mathrm{GFP}^{+}$single cells. 
In situ tumor induction was performed by subcutaneously injecting $10^{5}$ or $10^{6}$ NSCLC cells of specific genotypes into the flanks of nude mice (Mus musculus, nu/nu, 4- to 6-wk-old females; Charles River Laboratories). Animals were monitored for tumor growth by physical examination or luciferase imaging with IVIS (Xenogen) after intraperitoneal injection of beetle luciferin (Promega, Caliper) at $165 \mathrm{mg} / \mathrm{kg}$. At the endpoint of the experiments, tumors were harvested for molecular biology and/or histology.

All animal work were performed under the guidelines of the Division of Comparative Medicine (DCM) with protocols (0911098-11 and 0911-098-14) approved by the Massachusetts Institute of Technology Committee for Animal Care (CAC) and were consistent with the Guide for Care and Use of Laboratory Animals, National Research Council, 1996 (institutional animal welfare assurance no. A-3125-01).

\section{Tumor collection}

Tumor samples were harvested at the experimental end points. Briefly, Hypoxyprobe (pimonidazole $\mathrm{HCl}$ ) at a dose of $60 \mathrm{mg} / \mathrm{kg}$ body weight was intraperitoneally injected into mice. At $1 \mathrm{~h}$ after injection, the mice were sacrificed by lethal dose of $\mathrm{CO}_{2}$. Tumor tissues were immediately dissected. A fraction of tumor tissues was frozen on dry ice and stored at $-80^{\circ} \mathrm{C}$ for molecular analyses. Other fractions of the tissues were immediately fixed with $4 \%$ paraformaldehyde (PFA) overnight at $4^{\circ} \mathrm{C}$, followed by $75 \%$ ethanol wash, paraffin-embedding, and sectioning. Slides were subjected to histology analysis.

\section{IF and IHC}

Paraffin-embedded tumor tissues were sectioned at the thickness of $5 \mathrm{~mm}$. For general histopathological evaluation, tissue sections were stained with the standard hematoxylin-eosin (H\&E) method (Xue et al. 2010, 2012). Tissue sections were blocked with $3 \%$ goat serum in PBS for $30 \mathrm{~min}$ and incubated with single or multiple primary antibodies overnight at $4^{\circ} \mathrm{C}$. Primary antibodies included CD31 (BD), MAb1 (1:100; Hypoxyprobe, Inc.), and biotin-conjugated Isolectin B4 (1:400; Vector Laboratories). After washing with PBS, secondary antibodies were added to the sections and incubated for $1 \mathrm{~h}$ at room temperature. Secondary antibodies included an anti-rat Alexa 488 antibody (Invitrogen), an avidin-conjugated Alexa 555 antibody (Invitrogen), an anti-mouse Alexa 488, antibody (Invitrogen), and/or an avidin-conjugated horseradish peroxidase (HRP) antibody (Vector Laboratories). Sections were washed with PBS, followed by counterstaining with DAPI(Vector Laboratories), and were mounted in Vectashield mounting medium (Vector Laboratories). Some of the sections were counterstained with DAB chromogen (Invitrogen). Stained tissue samples were analyzed using a Zeiss confocal LSM700 microscope, a conventional light microscope, or an EVOS microscope (Advanced Microscope Group).

Whole-mount staining was conducted according to published methods (Xue et al. 2010, 2012). Briefly, tumor tissues were prepared into $\sim 2$-mm slices and washed in PBS for $1 \mathrm{~h}$, followed by incubation with $20 \mathrm{mg} / \mathrm{mL}$ proteinase $\mathrm{K}$ for $5 \mathrm{~min}$. The tissues were further permeabilized with methanol for $30 \mathrm{~min}$ and blocked with $3 \%$ fat-free milk in PBS, respectively. Tumor tissues were stained with primary antibody against CD31 overnight at $4^{\circ} \mathrm{C}$. After rigorous washing with PBS, the tissues were stained with anti-rat Alexa 488-conjugated secondary antibody for $2 \mathrm{~h}$ at room temperature. Stained slides were mounted in Vectashield mounting medium and imaged under a Zeiss confocal LSM700 microscope. Three-dimensional images of each sample were projected by acquiring seven layers of images at a 5 - $\mathrm{mm}$ distance between layers. Quantitative analyses from nine different tissue sections were performed.

IF and IHC slides were quantified in a blind selection of images using CellProfiler (Lamprecht et al. 2007), with pipeline implementation using custom python scripts, ImageJ (Schneider et al. 2012), or the color range and histogram tools from Adobe Photoshop CS.

\section{Tumor grow rate analysis}

Tumor induction was performed by subcutaneous injection of $10^{5}$ NSCLC cells of the desired genotype to 6 - to $\sim 8$-wk-old female nude mice ( $n=4$ each group). Tumors were measured over time by calipers, and volumes were estimated as spheroids using the formula $V=\pi \times a \times b \times h / 6$, where $V, a, b$, and $h$ are volume, length, width, and depth of a tumor, respectively.

\section{Standard molecular biology}

All conventional molecular biology experiments, including DNA/ RNA/protein extraction, PCR, RT-PCR, Western blot, and Northern blot were performed using standard molecular biology protocols with slight modifications or using commercially available kits (Promega, Qiagen, Zymo, Life Technologies, and TakaraBio). Proteins were quantified with Bradford assay (Fisher) and/or BCA assay (Fisher), calibrated by BSA standard curves, and normalized before quantification by Western or ELISA. Antibodies were from various sources, including Dicer (Cell Signaling), Vinculin (Sharp Laboratory), CD31 (BD), and FIH1 (SCBT). VEGF ELISA and angiogenesis protein array (R\&D Systems) were performed according to the manufacturer's protocols.

\section{MicroRNA Northern blot}

Total RNA was prepared from $1 \times 10^{6}$ to $\sim 5 \times 10^{6}$ cells or $50 \sim 200 \mathrm{mg}$ of tumor tissues using Trizol reagent (Life Technologies) according to the manufacturer's protocol. Five micrograms of RNA was mixed with an equal volume of formamide loading buffer, denatured for $5 \mathrm{~min}$ at $95^{\circ} \mathrm{C}$, and run for $1 \mathrm{~h}$ at $35 \mathrm{~W}$ on $8 \%$ or $12 \%$ denaturing polyacrylamide gels (Sequagel, National Diagnostics) after $30 \mathrm{~min}$ of prerunning. A semidry transfer apparatus set to a $300-\mathrm{mA}$ limit was used to transfer the RNA to a Hybond- $\mathrm{N}^{+}$nylon membrane (GE Healthcare Life Sciences) for $1.5 \mathrm{~h}$. Membrane containing RNA was then UV-cross-linked at $1.2 \times 10^{5} \mu \mathrm{J}$ in a Cross-linker 2400 (Stratagene) on top of Whatman paper. The membrane was prehybridized with Ultrahyb buffer (Ambion) for $0.5 \mathrm{~h}$ and then probed overnight at $42^{\circ} \mathrm{C}$ with $\left[\gamma^{-}{ }^{32} \mathrm{P}\right]$-ATP $5^{\prime}$ end-labeled DNA probes (IDT) reverse complementary to mature microRNA sequences. The membrane was washed twice for $30 \mathrm{~min}$ in $2 \times \mathrm{SSC} / 0.1 \%$ SDS buffer and then exposed to films (GE) for 1, 24, or $48 \mathrm{~h}$ and imaged on a Typhoon PhosphorImager (Molecular Dynamics). DNA oligo probes for U1 snRNA or U6 snRNA were used as loading controls.

\section{Oxygen conditioning and hypoxia setting}

Oxygen conditioning was performed similar to previously described methods (Metallo et al. 2012). Briefly, a hypoxia chamber was setup with oxygen, carbon dioxide, and nitrogen to adjust oxygen levels. Hypoxia condition was set to $1 \%$ oxygen and $5 \%$ carbon dioxide unless otherwise noted.

\section{Luciferase assay}

HIF transcription activity reporter FHRE-luc vector was ordered from Addgene (Brunet et al. 1999). Cells were cotransfected with FHRE-luc, pRL-CMV, and pCMV-GFP with/without additional constructs such as overexpression vectors or microRNAs. 
MicroRNAs (mmu-let-7-g-5p, mmu-mir-125-b-5p, and mmu-mir16-5p) for transfection were synthesized as customized siRNA (Dharmacon) based on the mature microRNA sequences from miRbase release 19, with a UU overhang at the 3' end. Dharmacon nontargeting control siRNA was used as a negative control. siRNAs and microRNAs were transfected at 10 50 $\mu \mathrm{M}$ concentrations. Human FIH1 cDNA constructs that overexpress FIH1 or FIH1-GFP were ordered from ThermoScientific and Addgene (21399 and 21403) (Metzen et al. 2003) and transfected at $1 \mu \mathrm{g} / \mathrm{mL}$. Human HIF1A cDNA construct was purchased from Thermo, and the N803A mutation was generated by oligo-based site-directed mutagenesis. Cells were lysed and assayed using a dual-luciferase kit (Promega). Data were first normalized to the constitutive Renilla luciferase and then to the control group under hypoxia.

\section{Expression profiling by $m R N A$-seq}

Total RNAs were prepared from $1 \times 10^{6}$ to $\sim 5 \times 10^{6}$ cells of specific genotypes (Dicer1 heterozygous or knockout) samples using Trizol reagent (Life Technologies). Samples were prequality-controlled on BioAnalyzer to ensure RNA integrity. Before library prep, $1 \mu \mathrm{L}$ of 1:50 diluted ERCC spike-in RNA (Life Technologies) was added to $1 \mu \mathrm{g}$ of total RNA. Normalized spiked total RNA samples were then used to generate highthroughput sequencing libraries using the Tru-seq kit (Illumina) following the manufacturer's instructions. Briefly, mRNAs were poly-A-purified from total RNAs, fragmented, and converted to cDNA using the dUTP second strand marking protocol outlined in Levin et al. (2010), with slight modifications (first strand synthesis incubation: $10 \mathrm{~min}$ at $25^{\circ} \mathrm{C}, 50 \mathrm{~min}$ at $42^{\circ} \mathrm{C}, 15 \mathrm{~min}$ at $70^{\circ} \mathrm{C}$, and $4^{\circ} \mathrm{C}$ hold; second strand synthesis incubation: $1 \mathrm{~h}$ at $16^{\circ} \mathrm{C}$ ). Synthesized cDNA samples were cleaned up with a $1.5 \times$ SPRI reaction, eluted in $50 \mu \mathrm{L}$ of EB, quality-controlled with the Bioanalyzer, and then adaptor-ligated and size-selected using automated SPRIworks system (Beckman Coulter) to generate Illumina libraries with a size range of $200-400 \mathrm{bp}$. The libraries were amplified using the primers against the Illumina adapters PE1.0 and/or PE2.0 (PE1.0, 5' -AGATCGGAAGAGCGGTTCAG CAGGAATGCCGAGACCG; and PE2.0, 5'-AGATCGGAAGA GCGTCGTGTAGGGAAAGAGTGT).

The libraries were uniquely barcoded to each sample during the amplification step and then used to generate clustered flow cells and sequenced on a HiSeq-2000 machine.

\section{Small RNA-seq (microRNA-seq)}

MicroRNA-seq samples were prepped from a subset of matched samples of the mRNA-seq RNA samples. Library preparation was performed using the small RNA library preparation kit (E7330, New England Biolabs) with size selection for 15 90 bp, a range including all potential mature microRNAs and precursors. Subsequent quality control (QC) sequencing steps were performed similarly following Illumina's instructions.

\section{mRNA and microRNRA sequencing data processing}

Raw fastq data of mRNA-seq were preprocessed using standard protocols.

Briefly, multiplexed barcoded reads from single-end or pairedend sequencing FASTQ files were bucketed by sample identity, and reads from both ends were processed for adapter removal using the fastx clipper utility from the Hannon laboratory FASTX-Toolkit suite (http://hannonlab.cshl.edu/fastx_toolkit). The adapters used were PE1.0 and/or PE2.0 as above. For pairedend reads, in order to eliminate reads from the low end of the distribution of insert sizes and limit the number of short sequences contributing to mapping ambiguity, both ends of a read were dropped if one (or both) of the reads resulted in a sequence $<15 \mathrm{bp}$ after adapter stripping. The distribution of insert lengths was determined empirically by aligning 1.25 million reads from each sample against the mouse transcriptome (University of California at Santa Cruz [UCSC] build $\mathrm{mm}$ 9) using the Bowtie short read alignment tool (Langmead et al. 2009). All libraries passed all initial QCs and mapping QCs.

Processed reads of mRNA-seq were aligned to the UCSC transcriptome and then to the genome (UCSC build mm9 unless otherwise noted) using the TopHat spliced junction alignment tool (Trapnell et al. 2009). Processed reads of microRNA-seq were first mapped to mouse mature microRNA sequences annotated in miRBase release 19, allowing for unique and repeat alignments with up to a single base pair mismatch per alignment. Reads that did not align to mature microRNA sequences were similarly mapped to microRNA hairpin sequences where possible, allowing for up to two mismatches per alignment. Mapping files (bam format) were used to quantify the relative gene expression level. In mRNA-seq, measurement was done in terms of reads per fragment per kilobase of transcript per million mapped reads (RPKM/FPKM) using Cufflinks (Trapnell et al. 2010). In microRNA-seq, measurement was done in reads per million of mapped reads (RPM) using custom scripts. Gene differential expression analysis was performed using cuffdiff (Trapnell et al. 2010) as well as ANOVA using custom R scripts.

\section{Gene set analyses}

Gene and genomic annotation was based on mouse genome UCSC build $\mathrm{mm} 9$ unless otherwise specified. Expressed genes were classified using an FPKM cutoff based on the overall distribution. Genes associated with certain biological processes were retrieved from the Gene Ontology database (http://www. geneontology.org). Gene ontology and gene set enrichment analyses were performed in DAVID (Huang et al. 2009), Ingenuity Pathway Analyses (IPA; Ingenuity Systems), and/or BioConductor (http://www.bioconductor.org). Transcription factor-target gene relationship data were retrieved from the literature knowledge base of the IPA and TRANSFAC databases (Matys et al. 2003).

The HIF antagonists were curated from the literature (Semenza 2003; Kaelin 2008) plus the IPA knowledgebase. The FIH1sensitive and FIH1-insensitive gene sets were curated from Dayan et al. (2006). Notch signaling pathway genes were retrieved from MSigdb (Liberzon et al. 2011).

\section{MicroRNA target gene analyses}

The microRNA family and sequences were retrieved from TargetScan 6.2 (Lewis et al. 2005) and miRbase 19 (Ambros et al. 2003). Cross-reference between TargetScan and miRbase was performed using custom scripts. Predicted microRNA target gene sets were retrieved from TargetScan 6.2. MicroRNA target gene differential expression analyses were carried out using customized scripts to intersect mRNA-seq data, microRNA expression data, and TargetScan prediction. For each microRNA, its target gene set included all annotated genes that had the particular seed sequence at the 3' UTR (TargetScan).

\section{Accession}

Illumina sequencing data have been deposited to Gene Expression Omnibus (accession no. GSE57043) and NCBI Sequence Read Archive (accession no. PRJNA244460).

\section{Acknowledgments}

We thank M. Lindstrom, M. Siafaca, A. Gurtan, A. Ravi, H. Yin, W. Isrealsen, M. vander Heiden, T. Tammela, W. Xue, M. Kumar, 
T. Jacks, and many other colleagues for their assistance. We thank all Sharp laboratory members for discussion. We thank G. Paradis, M. Jennings, S. Malstrom, M. Crowley, S. Levine, S. Motorola, D. Crowley, K. Cormier, C. Whittaker, W. Salmon, and other members in the Swanson Biotechnology Center and Core Facilities of Whitehead Institute. We thank E. Metzen for sharing FIH1 plasmids. We dedicate this paper to Sean Collier for his caring service and sacrifice. This work was made possible by grant number R01-CA133404 from the National Institutes of Health, by Massachusetts Institute of Technology-Harvard Center for Cancer Nanotechnology Excellence Grant U54 CA151884 from the National Cancer Institute, by a generous gift from the Marie D. and Pierre Casimir-Lambert Fund to P.A.S., partially by Koch Institute Support (core) grant P30-CA14051 from the National Cancer Institute, and in part by National Institutes of Health grants EB016101-01A1 and EB006365 to R.L. S.C. is a Damon Runyon Cancer Research Fellow (DRG-2117-12). X.W. is a Howard Hughes Medical Institute International Student Research Fellow. Y.X. is supported by the Swedish Research Council.

\section{References}

Ambros V, Bartel B, Bartel DP, Burge CB, Carrington JC, Chen X, Dreyfuss G, Eddy SR, Griffiths-Jones S, Marshall M, et al. 2003. A uniform system for microRNA annotation. RNA 9: 277-279.

Bartel DP. 2009. MicroRNAs: target recognition and regulatory functions. Cell 136: 215-233.

Bernstein E, Kim SY, Carmell MA, Murchison EP, Alcorn H, Li MZ, Mills AA, Elledge SI, Anderson KV, Hannon GJ. 2003. Dicer is essential for mouse development. Nat Genet 35: 215-217.

Brunet A, Bonni A, Zigmond MJ, Lin MZ, Juo P, Hu LS, Anderson MJ, Arden KC, Blenis J, Greenberg ME. 1999. Akt promotes cell survival by phosphorylating and inhibiting a Forkhead transcription factor. Cell 96: 857-868.

Calin GA, Croce CM. 2006. MicroRNA-cancer connection: the beginning of a new tale. Cancer Res 66: 7390-7394.

Carmeliet P, Jain RK. 2000. Angiogenesis in cancer and other diseases. Nature 407: 249-257.

Chen K, Wallis JW, McLellan MD, Larson DE, Kalicki JM, Pohl CS, McGrath SD, Wendl MC, Zhang Q, Locke DP, et al. 2009. BreakDancer: an algorithm for high-resolution mapping of genomic structural variation. Nat Methods 6: 677-681.

Chen Z, Lai TC, Jan YH, Lin FM, Wang WC, Xiao H, Wang YT, Sun W, Cui X, Li YS, et al. 2013. Hypoxia-responsive miRNAs target argonaute 1 to promote angiogenesis. J Clin Invest 123: 1057-1067.

Cimmino A, Calin GA, Fabbri M, Iorio MV, Ferracin M, Shimizu M, Wojcik SE, Aqeilan RI, Zupo S, Dono M, et al. 2005. miR-15 and miR-16 induce apoptosis by targeting BCL2. Proc Nat1 Acad Sci 102: 13944-13949.

Cong L, Ran FA, Cox D, Lin S, Barretto R, Habib N, Hsu PD, Wu $\mathrm{X}$, Jiang W, Marraffini LA, et al. 2013. Multiplex genome engineering using CRISPR/Cas systems. Science 339: 819-823.

Dayan F, Roux D, Brahimi-Horn MC, Pouyssegur J, Mazure NM. 2006. The oxygen sensor factor-inhibiting hypoxia-inducible factor-1 controls expression of distinct genes through the bifunctional transcriptional character of hypoxia-inducible factor-1 $\alpha$. Cancer Res 66: 3688-3698.

DuPage M, Dooley AL, Jacks T. 2009. Conditional mouse lung cancer models using adenoviral or lentiviral delivery of Cre recombinase. Nat Protoc 4: 1064-1072.

Ebert MS, Sharp PA. 2012. Roles for microRNAs in conferring robustness to biological processes. Cell 149: 515-524.

Fasanaro P, D'Alessandra Y, Di Stefano V, Melchionna R, Romani S, Pompilio G, Capogrossi MC, Martelli F. 2008. MicroRNA-210 modulates endothelial cell response to hypoxia and inhibits the receptor tyrosine kinase ligand Ephrin-A3. J Biol Chem 283: 15878-15883.

Friedman RC, Farh KK, Burge CB, Bartel DP. 2009. Most mammalian mRNAs are conserved targets of microRNAs. Genome Res 19: 92-105.

Gurtan AM, Lu V, Bhutkar A, Sharp PA. 2012. In vivo structurefunction analysis of human Dicer reveals directional processing of precursor miRNAs. RNA 18: 1116-1122.

Gurtan AM, Ravi A, Rahl PB, Bosson AD, InBaptiste CK, Bhutkar A, Whittaker CA, Young RA, Sharp PA. 2013. Let-7 represses Nr6al and a mid-gestation developmental program in adult fibroblasts. Genes Dev 27: 941-954.

He L, Thomson JM, Hemann MT, Hernando-Monge E, Mu D, Goodson S, Powers S, Cordon-Cardo C, Lowe SW, Hannon GJ, et al. 2005. A microRNA polycistron as a potential human oncogene. Nature 435: 828-833.

Heravi-Moussavi A, Anglesio MS, Cheng SW, Senz J, Yang W, Prentice L, Fejes AP, Chow C, Tone A, Kalloger SE, et al. 2012. Recurrent somatic DICER1 mutations in nonepithelial ovarian cancers. N Engl J Med 366: 234-242.

Hsu PD, Scott DA, Weinstein JA, Ran FA, Konermann S, Agarwala V, Li Y, Fine EJ, Wu X, Shalem O, et al. 2013. DNA targeting specificity of RNA-guided Cas9 nucleases. Nat Biotechnol 31: 827-832.

Hua Z, Lv Q, Ye W, Wong CK, Cai G, Gu D, Ji Y, Zhao C, Wang J, Yang BB, et al. 2006. MiRNA-directed regulation of VEGF and other angiogenic factors under hypoxia. PLOS ONE 1: e116.

Huang DW, Sherman BT, Lempicki RA. 2009. Systematic and integrative analysis of large gene lists using DAVID bioinformatics resources. Nat Protoc 4: 44-57.

Janke K, Brockmeier U, Kuhlmann K, Eisenacher M, Nolde J, Meyer HE, Mairbaurl H, Metzen E. 2013. Factor inhibiting HIF1 (FIH-1) modulates protein interactions of apoptosis-stimulating p53 binding protein 2 (ASPP2). J Cell Sci 126: 2629-2640.

Kaelin WG Jr. 2008. The von Hippel-Lindau tumour suppressor protein: $\mathrm{O}_{2}$ sensing and cancer. Nat Rev Cancer 8: 865-873.

Kaelin WG, Ratcliffe PJ. 2008. Oxygen sensing by metazoans: the central role of the HIF hydroxylase pathway. Mol Cell 30: 393-402.

Kelly TJ, Souza AL, Clish CB, Puigserver P. 2011. A hypoxiainduced positive feedback loop promotes hypoxia-inducible factor $1 \alpha$ stability through miR-210 suppression of glycerol3-phosphate dehydrogenase 1-like. Mol Cell Biol 31: 2696-2706.

Koboldt DC, Zhang Q, Larson DE, Shen D, McLellan MD, Lin L, Miller CA, Mardis ER, Ding L, Wilson RK. 2012. VarScan 2: somatic mutation and copy number alteration discovery in cancer by exome sequencing. Genome Res 22: 568-576.

Koivunen P, Hirsila M, Gunzler V, Kivirikko KI, Myllyharju J. 2004. Catalytic properties of the asparaginyl hydroxylase $(\mathrm{FIH})$ in the oxygen sensing pathway are distinct from those of its prolyl 4-hydroxylases. I Biol Chem 279: 9899-9904.

Konisti S, Kiriakidis S, Paleolog EM. 2012. Hypoxia-a key regulator of angiogenesis and inflammation in rheumatoid arthritis. Nat Rev Rheumatol 8: 153-162.

Kuehbacher A, Urbich C, Zeiher AM, Dimmeler S. 2007. Role of Dicer and Drosha for endothelial microRNA expression and angiogenesis. Circ Res 101: 59-68.

Kumar MS, Lu J, Mercer KL, Golub TR, Jacks T. 2007. Impaired microRNA processing enhances cellular transformation and tumorigenesis. Nat Genet 39: 673-677.

Kumar MS, Erkeland SJ, Pester RE, Chen CY, Ebert MS, Sharp PA, Jacks T. 2008. Suppression of non-small cell lung tumor development by the let-7 microRNA family. Proc Natl Acad Sci 105: 3903-3908.

Kumar MS, Pester RE, Chen CY, Lane K, Chin C, Lu J, Kirsch DG, Golub TR, Jacks T. 2009. Dicer1 functions as a haploinsufficient tumor suppressor. Genes Dev 23: 2700-2704. 
Lamprecht MR, Sabatini DM, Carpenter AE. 2007. CellProfiler: free, versatile software for automated biological image analysis. Biotechniques 42: 71-75.

Lando D, Peet DI, Gorman JJ, Whelan DA, Whitelaw ML, Bruick RK. 2002. FIH-1 is an asparaginyl hydroxylase enzyme that regulates the transcriptional activity of hypoxia-inducible factor. Genes Dev 16: 1466-1471.

Langmead B, Trapnell C, Pop M, Salzberg SL. 2009. Ultrafast and memory-efficient alignment of short DNA sequences to the human genome. Genome Biol 10: R25.

Leung DW, Cachianes G, Kuang WJ, Goeddel DV, Ferrara N. 1989. Vascular endothelial growth factor is a secreted angiogenic mitogen. Science 246: 1306-1309.

Levin JZ, Yassour M, Adiconis X, Nusbaum C, Thompson DA, Friedman N, Gnirke A, Regev A. 2010. Comprehensive comparative analysis of strand-specific RNA sequencing methods. Nat Methods 7: 709-715.

Lewis BP, Burge CB, Bartel DP. 2005. Conserved seed pairing, often flanked by adenosines, indicates that thousands of human genes are microRNA targets. Cell 120: 15-20.

Liberzon A, Subramanian A, Pinchback R, Thorvaldsdottir H, Tamayo P, Mesirov JP. 2011. Molecular signatures database (MSigDB) 3.0. Bioinformatics 27: 1739-1740.

Liu CJ, Tsai MM, Hung PS, Kao SY, Liu TY, Wu KJ, Chiou SH, Lin SC, Chang KW. 2010. miR-31 ablates expression of the HIF regulatory factor FIH to activate the HIF pathway in head and neck carcinoma. Cancer Res 70: 1635-1644.

Lu J, Getz G, Miska EA, Alvarez-Saavedra E, Lamb J, Peck D, Sweet-Cordero A, Ebert BL, Mak RH, Ferrando AA, et al. 2005. MicroRNA expression profiles classify human cancers. Nature 435: 834-838.

Mahon PC, Hirota K, Semenza GL. 2001. FIH-1: a novel protein that interacts with HIF- $1 \alpha$ and VHL to mediate repression of HIF-1 transcriptional activity. Genes Dev 15: 2675-2686.

Mali P, Yang LH, Esvelt KM, Aach J, Guell M, DiCarlo JE, Norville JE, Church GM. 2013. RNA-guided human genome engineering via Cas9. Science 339: 823-826.

Matys V, Fricke E, Geffers R, Gossling E, Haubrock M, Hehl R, Hornischer K, Karas D, Kel AE, Kel-Margoulis OV, et al. 2003. TRANSFAC: transcriptional regulation, from patterns to profiles. Nucleic Acids Res 31: 374-378.

McNeill LA, Hewitson KS, Claridge TD, Seibel JF, Horsfall LE, Schofield CJ. 2002. Hypoxia-inducible factor asparaginyl hydroxylase (FIH-1) catalyses hydroxylation at the $\beta$-carbon of asparagine-803. Biochem J 367: 571-575.

Metallo CM, Gameiro PA, Bell EL, Mattaini KR, Yang J, Hiller K, Jewell CM, Johnson ZR, Irvine DJ, Guarente L, et al. 2012. Reductive glutamine metabolism by IDH1 mediates lipogenesis under hypoxia. Nature 481: 380-384.

Metzen E, Berchner-Pfannschmidt U, Stengel P, Marxsen JH, Stolze I, Klinger M, Huang WQ, Wotzlaw C, Hellwig-Burgel $\mathrm{T}$, Jelkmann W, et al. 2003. Intracellular localisation of human HIF- $1 \alpha$ hydroxylases: implications for oxygen sensing. J Cell Sci 116: 1319-1326.

Nittner D, Lambertz I, Clermont F, Mestdagh P, Kohler C, Nielsen SJ, Jochemsen A, Speleman F, Vandesompele J, Dyer MA, et al. 2012. Synthetic lethality between Rb, p53 and Dicer or miR-17-92 in retinal progenitors suppresses retinoblastoma formation. Nat Cell Biol 14: 958-965.

Raleigh JA, Dewhirst MW, Thrall DE. 1996. Measuring tumor hypoxia. Semin Radiat Oncol 6: 37-45.

Ravi A, Gurtan AM, Kumar MS, Bhutkar A, Chin C, Lu V, Lees JA, Jacks T, Sharp PA. 2012. Proliferation and tumorigenesis of a murine sarcoma cell line in the absence of DICER1. Cancer Cell 21: 848-855.
Schneider CA, Rasband WS, Eliceiri KW. 2012. NIH Image to ImageJ: 25 years of image analysis. Nat Methods 9: 671-675.

Schofield CJ, Ratcliffe PJ. 2004. Oxygen sensing by HIF hydroxylases. Nat Rev Mol Cell Biol 5: 343-354.

Semenza GL. 2003. Targeting HIF-1 for cancer therapy. Nat Rev Cancer 3: 721-732.

Shen J, Xia W, Khotskaya YB, Huo L, Nakanishi K, Lim SO, Du Y, Wang $\mathrm{Y}$, Chang $\mathrm{WC}$, Chen $\mathrm{CH}$, et al. 2013. EGFR modulates microRNA maturation in response to hypoxia through phosphorylation of AGO2. Nature 497: 383-387.

Suarez Y, Sessa WC. 2009. MicroRNAs as novel regulators of angiogenesis. Circ Res 104: 442-454.

Suarez Y, Fernandez-Hernando C, Yu J, Gerber SA, Harrison KD, Pober JS, Iruela-Arispe ML, Merkenschlager M, Sessa WC. 2008. Dicer-dependent endothelial microRNAs are necessary for postnatal angiogenesis. Proc Natl Acad Sci 105: 14082-14087.

Takamizawa J, Konishi H, Yanagisawa K, Tomida S, Osada H, Endoh H, Harano T, Yatabe Y, Nagino M, Nimura Y, et al. 2004. Reduced expression of the let-7 microRNAs in human lung cancers in association with shortened postoperative survival. Cancer Res 64: 3753-3756.

Trapnell C, Pachter L, Salzberg SL. 2009. TopHat: discovering splice junctions with RNA-Seq. Bioinformatics 25: 1105-1111.

Trapnell C, Williams BA, Pertea G, Mortazavi A, Kwan G, van Baren MJ, Salzberg SL, Wold BJ, Pachter L. 2010. Transcript assembly and quantification by RNA-seq reveals unannotated transcripts and isoform switching during cell differentiation. Nat Biotechnol 28: 511-515.

Varghese AJ, Gulyas S, Mohindra JK. 1976. Hypoxia-dependent reduction of 1-(2-nitro-1-imidazolyl)-3-methoxy-2-propanol by Chinese hamster ovary cells and KHT tumor cells in vitro and in vivo. Cancer Res 36: 3761-3765.

Wang J, Wang Y, Ma Y, Lan Y, Yang X. 2013. Transforming growth factor $\beta$-regulated microRNA-29a promotes angiogenesis through targeting the phosphatase and tensin homolog in endothelium. J Biol Chem 288: 10418-10426.

Weinberg RA. 2007. The biology of cancer. Garland Science, New York.

Wilkins SE, Hyvarinen J, Chicher J, Gorman J, Peet DJ, Bilton RL, Koivunen P. 2009. Differences in hydroxylation and binding of Notch and HIF-1 $\alpha$ demonstrate substrate selectivity for factor inhibiting HIF-1 (FIH-1). Int I Biochem Cell Biol 41: 1563-1571.

Xue Y, Lim S, Brakenhielm E, Cao YH. 2010. Adipose angiogenesis: quantitative methods to study microvessel growth, regression and remodeling in vivo. Nat Protoc 5: 912-920.

Xue Y, Lim S, Yang YL, Wang ZW, Jensen LDE, Hedlund EM, Andersson P, Sasahara M, Larsson O, Galter D, et al. 2012. PDGF-BB modulates hematopoiesis and tumor angiogenesis by inducing erythropoietin production in stromal cells. Nat Med 18: 100-110.

Yang WJ, Yang DD, Na SQ, Sandusky GE, Zhang Q, Zhao GS. 2005. Dicer is required for embryonic angiogenesis during mouse development. I Biol Chem 280: 9330-9335.

Zhang N, Fu ZX, Linke S, Chicher J, Gorman JJ, Visk D, Haddad GG, Poellinger L, Peet DJ, Powell F, et al. 2010. The asparaginyl hydroxylase factor inhibiting HIF-1 $\alpha$ is an essential regulator of metabolism. Cell Metab 11: 364-378.

Zheng X, Linke S, Dias JM, Gradin K, Wallis TP, Hamilton BR, Gustafsson M, Ruas JL, Wilkins S, Bilton RL, et al. 2008. Interaction with factor inhibiting HIF-1 defines an additional mode of cross-coupling between the Notch and hypoxia signaling pathways. Proc Natl Acad Sci 105: 3368-3373.

Zhuang G, Wu X, Jiang Z, Kasman I, Yao J, Guan Y, Oeh J, Modrusan Z, Bais C, Sampath D, et al. 2012. Tumour-secreted miR-9 promotes endothelial cell migration and angiogenesis by activating the JAK-STAT pathway. EMBO J 31: 3513-3523. 


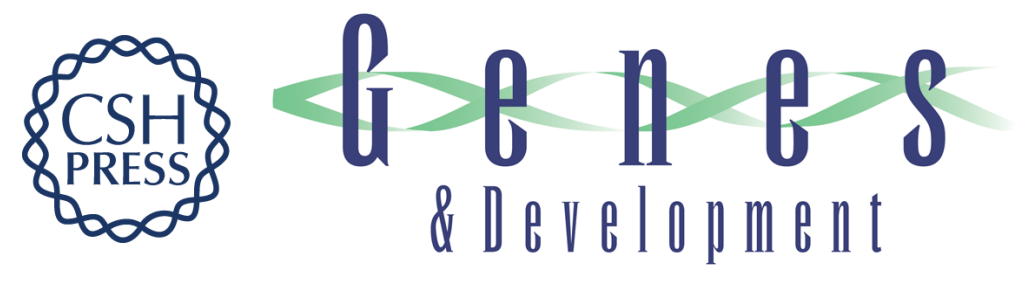

\section{Global microRNA depletion suppresses tumor angiogenesis}

Sidi Chen, Yuan Xue, Xuebing Wu, et al.

Genes Dev. 2014, 28: originally published online May 1, 2014

Access the most recent version at doi:10.1101/gad.239681.114

\section{Supplemental http://genesdev.cshlp.org/content/suppl/2014/04/25/gad.239681.114.DC1 \\ Material \\ References This article cites 69 articles, 30 of which can be accessed free at: http://genesdev.cshlp.org/content/28/10/1054.full.html\#ref-list-1 \\ Creative This article is distributed exclusively by Cold Spring Harbor Laboratory Press for the first Commons six months after the full-issue publication date (see \\ License http://genesdev.cshlp.org/site/misc/terms.xhtml). After six months, it is available under a Creative Commons License (Attribution-NonCommercial 4.0 International), as described at http://creativecommons.org/licenses/by-nc/4.0/.}

Email Alerting Receive free email alerts when new articles cite this article - sign up in the box at the top Service right corner of the article or click here.

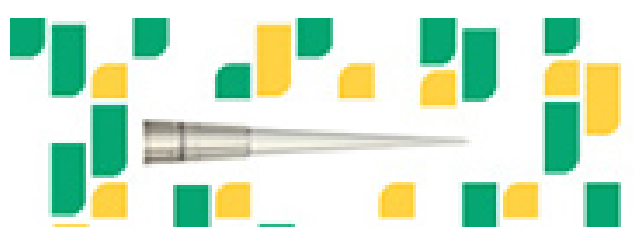

Focused on your science. 